JOURNAL OF THE

AMERICAN MATHEMATICAL SOCIETY

Volume 18, Number 4, Pages 919-941

S 0894-0347(05)00488-1

Article electronically published on May 25, 2005

\title{
HOMOLOGICAL METHODS FOR HYPERGEOMETRIC FAMILIES
}

\author{
LAURA FELICIA MATUSEVICH, EZRA MILLER, AND ULI WALTHER
}

Uli Walther dedicates this paper to the memory of his father, Hansjoachim Walther.

\section{Contents}

1. Introduction

2. Upper semi-continuity of rank in holonomic families

3. Rank-jumps as failures of flatness

4. Euler-Koszul homology of toric modules

5. Rigidity and holonomicity of Euler-Koszul homology

6. Euler-Koszul homology detects local cohomology

7. Global Euler-Koszul homology as a holonomic family

8. Isomorphism of the two homology theories

9. Combinatorics of hypergeometric ranks

Acknowledgments

References

\section{INTRODUCTION}

In the late 1980s, Gel'fand, Graev, and Zelevinskil introduced a class of systems of linear partial differential equations that are closely related to toric varieties GGZ87. These systems, now called GKZ systems, or A-hypergeometric systems, are constructed from a $d \times n$ integer matrix $A$ of rank $d$ and a complex parameter vector $\beta \in \mathbb{C}^{d}$, and are denoted by $H_{A}(\beta)$. We assume that the columns of $A$ lie in a single open halfspace. $A$-hypergeometric systems arise in various instances in algebraic geometry. For example, solutions of $A$-hypergeometric systems appear as toric residues CDS01, and special cases are mirror transforms of generating functions for intersection numbers on moduli spaces of curves [CK99],

Received by the editors June 22, 2004.

2000 Mathematics Subject Classification. Primary 13N10, 13D45, 14D99, 13F99, 16E99; Secondary 32C38, 35A27, 14M25, 70F20, 33C70, 13C14, $13 \mathrm{D} 07$.

Key words and phrases. Hypergeometric system, Cohen-Macaulay, toric, local cohomology, holonomic, $D$-module.

The first author was partially supported by a postdoctoral fellowship from MSRI and an NSF Postdoctoral Fellowship.

The second author was partially supported by NSF Grant DMS-0304789.

The third author was partially supported by the DfG, the Humboldt foundation, and NSF Grant DMS-0100509. 
the $A$-hypergeometric systems there being Picard-Fuchs equations governing the variation of Hodge structures for Calabi-Yau toric hypersurfaces.

The first fundamental results about the systems $H_{A}(\beta)$ were proved by Gel'fand, Graev, Kapranov, and Zelevinskil. These results concerned the case where the semigroup $\mathbb{N} A$ generated by the columns of $A$ gives rise to a semigroup ring $\mathbb{C}[\mathbb{N} A]$ that is Cohen-Macaulay and graded in the standard $\mathbb{Z}$-grading GGZ87, GKZ89. In geometric terms, the associated projective toric variety $X_{A}$ is arithmetically CohenMacaulay. The above authors showed that, in this case, the system $H_{A}(\beta)$ gives a holonomic module over the ring $D$ of polynomial $\mathbb{C}$-linear differential operators in $n$ variables, and hence $H_{A}(\beta)$ has finite rank; that is, the dimension of its space of holomorphic solutions is finite. Furthermore, they showed that this dimension can be expressed combinatorially, as the integer $\operatorname{vol}(A)$ that is $d$ ! times the Euclidean volume of the convex hull of the columns of $A$ and the origin $0 \in \mathbb{Z}^{d}$. The remarkable fact is that their rank formula holds independently of the parameter $\beta$.

Even if $\mathbb{C}[\mathbb{N} A]$ is not Cohen-Macaulay or $\mathbb{Z}$-graded, Adolphson showed in [Ado94] that $H_{A}(\beta)$ is always a holonomic ideal. He further proved that, for all parameters outside of a closed locally finite arrangement of countably many "semi-resonant" affine hyperplanes, the characterization of rank through volume is still correct.

It came as quite a surprise when in ST98 an example was given showing that if $\mathbb{C}[\mathbb{N} A]$ is not Cohen-Macaulay, then not all parameters $\beta$ have to give the same rank. One is hence prompted to introduce $\mathcal{E}_{A}$, the collection of those exceptional parameters $\beta \in \mathbb{C}^{d}$ for which the rank does not take the expected value. Nearly at the same time the case of projective toric curves was discussed completely in CDD99: the set $\mathcal{E}_{A}$ of exceptional parameters is finite in this case, and empty precisely when $\mathbb{C}[\mathbb{N} A]$ is Cohen-Macaulay; moreover, at each $\beta \in \mathcal{E}_{A}$ the rank exceeds the volume by exactly 1 . It was shown soon after in SST00 that the rank can never be smaller than the volume as long as $\mathbb{C}[\mathbb{N} A]$ is $\mathbb{Z}$-graded, and it was established in the same book that $\mathcal{E}_{A}$ is in fact contained in a finite affine subspace arrangement. More recently the much stronger fact emerged that $\mathcal{E}_{A}$ is a finite union of Zariski locally closed sets by means of Gröbner basis techniques Mat03. While rank-jumps can be arbitrarily large [MW04, the absence of rank-jumping parameters is equivalent to the Cohen-Macaulay property for $\mathbb{Z}$-graded $\mathbb{C}[\mathbb{N} A]$ when either $\mathbb{C}[\mathbb{N} A]$ has codimension two [Mat01, or if the convex hull of $A$ is a simplex Sai02, or if $\mathbb{C}[\mathbb{N} A]$ is a polynomial ring modulo a generic toric ideal Mat03.

Encouraged by these results, which suggest an algebraic structure on the set of exceptional parameters, it was conjectured in MM05 that the obstructions to the Cohen-Macaulayness of $\mathbb{C}[\mathbb{N} A]$ and the set of exceptional parameters are identified in an explicit manner. To be precise, let $H_{\mathfrak{m}}^{<d}(\mathbb{C}[\mathbb{N} A])$ be the direct sum of all the local cohomology modules supported at the maximal homogeneous ideal $\mathfrak{m}$ of $\mathbb{C}[\mathbb{N} A]$ in cohomological degrees less than $d$. Then define the set $E_{A}$ of exceptional quasi-degrees to be the Zariski closure in $\mathbb{C}^{d}$ of the set of $\mathbb{Z}^{d}$-graded degrees $\alpha$ such that $H_{\mathrm{m}}^{<d}(\mathbb{C}[\mathbb{N} A])$ has a nonzero element in degree $-\alpha$. With this notation, the motivating result in this article is the following.

Theorem 1.1. For any rank $d$ matrix $A \in \mathbb{Z}^{d \times n}$ as above, the set $\mathcal{E}_{A}$ of exceptional (that is, rank-jumping) parameters is identical to the set $E_{A}$ of exceptional quasidegrees.

We note that there is no assumption on $\mathbb{C}[\mathbb{N} A]$ being $\mathbb{Z}$-graded. The $\mathbb{Z}$-graded simplicial case of this result was proved in MM05] using results of [Sai02]. 
Methods and results. The first step in our proof of Theorem 1.1 is to construct a homological theory to systematically detect rank-jumps. In Sections 2 and 3 we study rank variation in any family of holonomic modules over any base $B$, and not just $A$-hypergeometric families over $B=\mathbb{C}^{d}$. The idea is that under a suitable coherence assumption (Definition 2.1), holonomic ranks behave like fiber dimensions in families of algebraic varieties. In particular, rank is constant almost everywhere and can only increase on closed subsets of $B$ (Theorem 2.6). We develop a computational tool to check for rank-jumps at a smooth point $\beta \in B$ : since the rank-jump occurs through a failure of flatness at $\beta$, ordinary Koszul homology detects it (Theorem 3.1 and Corollary 3.3).

The second step in proving Theorem 1.1 is to construct a homological theory for $D$-modules that reproduces the set $E_{A}$ of exceptional quasi-degrees, which a priori arises from the commutative notion of local cohomology. Our main observation along these lines is that the Euler-Koszul complex, which was already known to Gel'fand, Kapranov, and Zelevinskii for Cohen-Macaulay $\mathbb{Z}$-graded semigroup rings [GKZ89, generalizes to fill this need. Adolphson [Ado99] recognized that when the semigroup is not Cohen-Macaulay, certain conditions guarantee that this complex has zero homology. Here, we develop Euler-Koszul homology (Definition 4.2) for the class of toric modules (Definition 4.5), which are slight generalizations of $\mathbb{Z}^{d_{-}}$ graded modules over the semigroup ring $\mathbb{C}[\mathbb{N} A]$. For any toric module $M$, we show in Theorem 6.6 that the set of parameters $\beta$ for which the Euler-Koszul complex has nonzero higher homology is precisely the analogue for $M$ of the exceptional quasi-degree set $E_{A}$ defined above for $M=\mathbb{C}[\mathbb{N} A]$.

Having now two cohomology theories, one to recover the local cohomology quasidegrees for hypergeometric families, and another to detect rank-jumping parameters for general holonomic families, we link them in a central result of this article, Theorem 8.2 for toric modules, these two theories coincide. Consequently, we obtain Theorem 1.1 as the special case $M=\mathbb{C}[\mathbb{N} A]$ of Theorem 9.1, which holds for arbitrary toric modules $M$. We deduce in Corollary 9.2 the equivalence of the Cohen-Macaulay condition on $\mathbb{C}[\mathbb{N} A]$ with the absence of rank-jumps in the GKZ hypergeometric system $H_{A}(\beta)$.

As a final comment, let us note that we avoid the explicit computation of solutions to hypergeometric systems. This contrasts with the previously cited constructions of exceptional parameters, which rely on combinatorial techniques to produce solutions. It is for this reason that these constructions contained the assumption that the semigroup ring $\mathbb{C}[\mathbb{N} A]$ is graded in the usual $\mathbb{Z}$-grading, for this implies that the corresponding hypergeometric systems are regular holonomic and thus have solutions expressible as power series with logarithms, with all the combinatorial control this provides. Our use of homological techniques makes the results in this article valid in both the regular and nonregular cases.

\section{UPPER SEMI-CONTINUITY OF RANK IN HOLONOMIC FAMILIES}

The results in the first part of this article (Sections 2 and 3) deal with general modules over the Weyl algebra, without restricting to the hypergeometric realm. In this section we define the notion of a holonomic family of $D$-modules and show that the holonomic rank constitutes an upper semi-continuous function on such a family. 
Throughout this article, $\partial=\partial_{1}, \ldots, \partial_{n}$ refer to the partial derivation operators with respect to the variables $x=x_{1}, \ldots, x_{n}$. Writing $\delta_{i j}$ for the Kronecker delta, so that $\delta_{i j}=1$ if $i=j$ and $\delta_{i j}=0$ otherwise, the Weyl algebra $D$ is the quotient of the free associative $\mathbb{C}$-algebra on $\left\{x_{i}, \partial_{i}\right\}_{i=1}^{n}$ by the two-sided ideal

$$
\left\langle x_{i} x_{j}-x_{j} x_{i}, \partial_{i} \partial_{j}-\partial_{j} \partial_{i}, \partial_{i} x_{j}-x_{j} \partial_{i}-\delta_{i j}\right\rangle_{i, j=1}^{n} \text {. }
$$

Every left $D$-module is also a module over the commutative subalgebra $\mathbb{C}[x]$ of $D$. If $N$ is any $\mathbb{C}[x]$-module or sheaf of $\mathbb{C}[x]$-modules on some space, let $N(x)=$ $\mathbb{C}(x) \otimes_{\mathbb{C}[x]} N$ denote the localization by inverting all polynomials in the $x$-variables. We shall also have occasion to consider modules, sheaves of modules, and schemes defined over $\mathbb{C}$, and if $N$ is such an object, then $N(x)$ denotes its base extension to the field $\mathbb{C}(x)$ of rational functions.

Our focus is on holonomic $D$-modules, which are by definition finitely generated left $D$-modules $N$ such that $\operatorname{Ext}_{D}^{j}(N, D)=0$ whenever $j \neq n$. The holonomic modules form a full subcategory of the category of $D$-modules that is closed under taking extensions, submodules, and quotient modules. When $N$ is a holonomic module, the $\mathbb{C}(x)$-vector space $N(x)$ has finite dimension, and this dimension equals the holonomic rank $\operatorname{rank}(N)$ by a celebrated theorem of Kashiwara; see SST00, Thm. 1.4.19, Cor. 1.4.14]. We note that rank is hence additive in short exact sequences of holonomic modules.

We are interested in families of $D$-modules parameterized by a Noetherian complex algebraic variety $B$ with structure sheaf $\mathcal{O}_{B}$. If $\beta \in B$ we denote by $\mathfrak{p}_{\beta}$ the prime ideal (sheaf) of $\beta$, and by $\kappa_{\beta}$ the residue field of the stalk $\mathcal{O}_{B, \beta}$, so $\kappa_{\beta}=\mathcal{O}_{B, \beta} / \mathfrak{p}_{\beta} \mathcal{O}_{B, \beta}$.

Consider the sheaf $D \otimes_{\mathbb{C}} \mathcal{O}_{B}$ of noncommutative $\mathcal{O}_{B}$-algebras on $B$. By a coherent sheaf of left $\left(D \otimes_{\mathbb{C}} \mathcal{O}_{B}\right)$-modules we mean a quasi-coherent sheaf of $\mathcal{O}_{B}$-modules on $B$ whose sections over each open affine subset $U \subseteq B$ are finitely generated over the ring of global sections of $D \otimes_{\mathbb{C}} \mathcal{O}_{U}$. The sheaf $D \otimes_{\mathbb{C}} \mathcal{O}_{B}$ contains the subsheaf $\mathcal{O}_{B}[x]=\mathbb{C}[x] \otimes_{\mathbb{C}} \mathcal{O}_{B}$ of commutative polynomials over $\mathcal{O}_{B}$, whose localization at $\langle 0\rangle \in \operatorname{Spec}(\mathbb{C}[x])$ is by our conventions $\mathcal{O}_{B}(x)$. The sheaf-spectrum of $\mathcal{O}_{B}(x)$ is the base-extended scheme $B(x)=\operatorname{Spec} \mathbb{C}(x) \times_{\text {Spec } \mathbb{C}} B$, which can be considered as a scheme over $\mathbb{C}(x)$ or as fibered over $B$.

Definition 2.1. A holonomic family over a complex scheme $B$ is a coherent sheaf $\tilde{\mathcal{M}}$ of left $\left(D \otimes_{\mathbb{C}} \mathcal{O}_{B}\right)$-modules whose fibers $\mathcal{M}_{\beta}=\tilde{\mathcal{M}} \otimes_{\mathcal{O}_{B}} \kappa_{\beta}$ are holonomic $D$-modules for all $\beta \in B$, and whose rank sheaf $\tilde{\mathcal{M}}(x)$ is coherent on the scheme $B(x)$.

Note that each holonomic family over $B$ is generated by its global sections if $B$ is affine.

Example 2.2. When $B$ equals the complex vector space $\mathbb{C}^{d}$ of dimension $d$, the structure sheaf $\mathcal{O}_{B}$ has global sections $\mathbb{C}[b]$, the commutative polynomial ring in variables $b=b_{1}, \ldots, b_{d}$. A holonomic family $\tilde{\mathcal{M}}$ over $\mathbb{C}^{d}$ can be represented by its global sections, a left $D[b]$-module $\mathcal{M}$ with finitely many generators and relations. In order to be a holonomic family in our sense, $\mathcal{M}_{\beta}=\mathcal{M} \otimes_{\mathcal{O}_{B}} \kappa_{\beta}$ has to be a holonomic $D$-module for all $\beta \in \mathbb{C}^{d}$ while the global section module $\mathcal{M}(x)=$ $\mathbb{C}(x) \otimes_{\mathbb{C}[x]} \mathcal{M}$ of the rank sheaf has to be a finitely generated $\mathbb{C}[b](x)$-module.

The rank sheaf $\tilde{\mathcal{M}}(x)$ is a sheaf of $\mathcal{O}_{B}(x)$-modules on $B(x)$, but ignoring the process of pushing $\tilde{\mathcal{M}}(x)$ down to $B$ we abuse notation and speak of the fiber 
of $\tilde{\mathcal{M}}(x)$ over $\beta \in B$. Let $B_{\mathbb{C}} \subseteq B$ be the $\mathbb{C}$-valued closed points of $B$ (that is, points with residue field $\mathbb{C}$ ).

Proposition 2.3. For a holonomic family $\tilde{\mathcal{M}}$ over a scheme $B$, the fiber of $\tilde{\mathcal{M}}(x)$ over each point $\beta \in B$ is $\mathcal{M}_{\beta}(x)$, which is a $\mathbb{C}(x)$-vector space of dimension $\operatorname{rank}\left(\mathcal{M}_{\beta}\right)$ if $\beta \in B_{\mathbb{C}}$.

Proof. Tensoring with the rational functions $\mathbb{C}(x)$ over $\mathbb{C}[x]$ commutes with the passage from $\tilde{\mathcal{M}}$ to $\mathcal{M}_{\beta}=\tilde{\mathcal{M}} \otimes_{\mathcal{O}_{B}} \kappa_{\beta}$.

The next lemma says that Zariski closed subsets of the base-extended scheme $B(x)$ descend to Zariski closed subsets of the original scheme $B$. We use the term prime ideal to refer to the kernel of the morphism of structure sheaves associated to any map from the spectrum of a field to $B$.

Lemma 2.4. For a scheme $B$ defined over $\mathbb{C}$, the map taking each prime ideal $\mathfrak{p} \subset \mathcal{O}_{B}$ to its extension $\mathfrak{p} \mathcal{O}_{B}(x)$ constitutes a continuous injection $B \rightarrow B(x)$ of topological spaces.

Proof. Note first that if $\mathfrak{p} \subset \mathcal{O}_{B}$ is prime, then the extension $\mathfrak{p} \mathcal{O}_{B}(x)$ is prime. The lemma is equivalent to saying that, for every subset $Y \subseteq B(x)$ that is Zariski closed, the set of points $\beta \in B$ whose prime ideal sheaves $\mathfrak{p}_{\beta}$ in $\mathcal{O}_{B}$ extend to prime ideals $\mathfrak{p}_{\beta} \mathcal{O}_{B}(x) \in Y$ is Zariski closed in $B$. This statement is local on $B$, so we may assume that $B$ is affine.

Suppose that $Y$ is the variety of a set $\mathcal{F}$ of global sections of $\mathcal{O}_{B}(x)$. Any free $\mathbb{C}$-basis for $\mathbb{C}(x)$ is also a free $\mathcal{O}_{B}$-basis for $\mathcal{O}_{B}(x)$; choose such a basis. For each global section $f \in \mathcal{F}$, let $\mathcal{G}_{f} \subseteq \mathcal{O}_{B}$ be its set of nonzero coefficients in the chosen basis. Then a prime $\mathfrak{p} \subset \mathcal{O}_{B}$ satisfies $\mathfrak{p} \mathcal{O}_{B}(x) \in Y$ if and only if $\mathfrak{p}$ contains the sets $\mathcal{G}_{f}$ for all $f \in \mathcal{F}$.

Example 2.5. Lemma 2.4 does not say that $B$ is closed in $B(x)$; indeed, the closure of the image is all of $B(x)$, whereas there are always points in $B(x) \backslash B$ if $B$ has dimension $>0$. Neither does Lemma 2.4 say that the morphism $B(x) \rightarrow B$ takes closed sets to closed sets. For example, if $n=d=1$ in Example 2.2 then the variety of the ideal $\langle b-x\rangle \subset \mathbb{C}[b](x)$ is a Zariski closed point in $B(x)$, whereas the image of this point in $B=\mathbb{C}^{1}$ is the generic point. Note that the set of prime ideals $\mathfrak{p} \subset \mathbb{C}[b]$ whose extensions $\mathfrak{p} \mathbb{C}[b](x)$ contain $\langle b-x\rangle$ is empty and therefore Zariski closed.

We now come to the main result of this section. To state it, we call $\beta \in B$ rankjumping if (i) $\beta \in B_{\mathbb{C}}$ is a $\mathbb{C}$-valued point, and (ii) for all open sets $U$ containing $\beta$, the rank of $\mathcal{M}_{\beta}$ is strictly greater than the minimal holonomic rank attained by any fiber $\mathcal{M}_{\beta^{\prime}}$ for $\beta^{\prime} \in U_{\mathbb{C}}$.

Theorem 2.6. If $\mathcal{M}$ is a holonomic family over a scheme $B$, then the function $\beta \mapsto \operatorname{rank}\left(\mathcal{M}_{\beta}\right)$ is upper semi-continuous both on $B$ and on $B_{\mathbb{C}}$ (endowed with the subspace topology). In particular, the locus of rank-jumping points $\beta \in B_{\mathbb{C}}$ is closed in $B_{\mathbb{C}}$.

Proof. The function sending each point $\beta(x) \in B(x)$ to the $\mathbb{C}(x)$-dimension of the fiber $\tilde{\mathcal{M}}(x) \otimes \kappa_{\beta(x)}$ is upper semi-continuous on $B(x)$ because $\tilde{\mathcal{M}}(x)$ is coherent on $B(x)$; see [Har77, III.12.7.2]. Therefore, given an integer $i$, the subset of points in $B(x)$ on which this fiber dimension is at least $i$ is Zariski closed. Lemma 2.4 shows 
that the corresponding subset of points in $B$ is Zariski closed in $B$. Proposition 2.3 . which says that the fiber dimensions over $\beta \in B_{\mathbb{C}}$ are holonomic ranks, completes the proof.

Remark 2.7. Without the coherence hypothesis on $\tilde{\mathcal{M}}(x)$ over $B(x)$, the conclusion of Theorem 2.6 can be false, even if $B$ is of finite type over $\mathbb{C}$ and all of the fibers $\mathcal{M}_{\beta}$ for $\beta \in B_{\mathbb{C}}$ are holonomic. For an example, consider the setup in Example 2.2 with $n=d=1$, and take $\mathcal{M}=D[b] /\langle b x \partial-1\rangle$. When $\beta \neq\langle b-0\rangle$, the fiber over $\beta$ is the rank 1 holonomic module corresponding to the solution $x^{1 / \beta}$. But when $\beta=\langle b-0\rangle$ the fiber of $\tilde{\mathcal{M}}$ is zero. Hence the rank actually drops on the closed subset $\{0\} \subset \mathbb{C}^{1}$. See Example 3.5 for further details.

Remark 2.8. Fix a holonomic family $\tilde{\mathcal{M}}$. The semi-continuity of the holonomic rank in Theorem 2.6 suggests that a "solution sheaf" $\mathcal{S}$ ol $(\tilde{\mathcal{M}})$, constructed as below, might be an algebraic coherent sheaf on $B$; a priori, it can only be expected to be an analytic sheaf. See Remark 9.6 for further comments on this issue, as it relates to hypergeometric systems.

For the construction, suppose there is a vector $v \in \mathbb{C}^{n}$ such that the singular locus of $\mathcal{M}_{\beta}$ does not contain $v$ for any $\beta \in B_{\mathbb{C}}$ (this is certainly possible locally on $B)$. Let the point $v$ have maximal ideal $\left\langle x_{1}-v_{1}, \ldots, x_{n}-v_{n}\right\rangle$ in $\mathbb{C}[x]$. The $D$-module restriction of $\tilde{\mathcal{M}}_{\beta}$ to $v$ is the derived tensor product over $D$ with the right $D$-module $D /\left\langle x_{1}-v_{1}, \ldots, x_{n}-v_{n}\right\rangle D$. This restriction is naturally dual to the space of formal power series solutions of $\tilde{\mathcal{M}}_{\beta}$ at $v$. But as $v$ is a regular point, formal and convergent solutions of $\tilde{\mathcal{M}}_{\beta}$ are identical. Since tensor products commute in $x$ and $b$, restricting the family $\tilde{\mathcal{M}}$ gives rise to an $\mathcal{O}_{B}$-module whose fiber over each point $\beta \in B_{\mathbb{C}}$ is naturally dual to the solution space of $\tilde{\mathcal{M}}_{\beta}$. Taking the dual over $\mathcal{O}_{B}$, if it can be appropriately defined, would yield the analytic $\mathcal{O}_{B^{-}}$ module $\operatorname{Sol}(\tilde{\mathcal{M}})$.

\section{RANK-JUMPS AS FAILURES OF FLATNESS}

For applications to hypergeometric systems, we need some concrete criteria to help us apply the results of the previous section. First we characterize rank-jumps in holonomic families over reduced schemes using Koszul homology in a commutative setting (Corollary 3.3). Then we provide a criterion for a family of $D$-modules to satisfy the coherence property required of a holonomic family (Proposition 3.4); we will appeal to it in Section 7 .

In the following statement, we use the standard notion of reduced for a scheme to mean that the coordinate rings of its affine open subschemes have no nilpotent elements.

Theorem 3.1. Let $\tilde{\mathcal{M}}$ be a holonomic family over a reduced scheme $B$ of finite type over $\mathbb{C}$, and fix a $\mathbb{C}$-valued closed point $\beta \in B_{\mathbb{C}}$. The parameter $\beta$ is rank-jumping if and only if $\operatorname{Tor}_{i}^{\mathcal{O}_{B}}\left(\tilde{\mathcal{M}}(x), \kappa_{\beta}\right)$ is nonzero for some homological degree $i>0$.

Proof. Since $B$ is of finite type, the set $B_{\mathbb{C}}$ is dense in $B$. Via the inclusion of Lemma 2.4, $B$ is always dense in $B(x)$, so we deduce that $B_{\mathbb{C}}$ is also dense in $B(x)$. Therefore

$$
\min _{\beta \in B_{\mathbb{C}}}\left\{\operatorname{rank}\left(\mathcal{M}_{\beta}\right)\right\}=\min _{\beta \in B(x)}\left\{\operatorname{dim}_{\mathbb{C}(x)} \tilde{\mathcal{M}}_{\beta}(x)\right\}
$$


by Proposition 2.3 and the upper semi-continuity of the fiber dimension Har77, III.12.7.2]. By [Har77, Exercise II.5.8], the points $\beta(x) \in B(x)$ over which the fiber dimension equals the quantity given in (函) coincide with the points $\beta(x)$ near which $\tilde{\mathcal{M}}(x)$ is locally free. But local freeness and flatness agree for coherent sheaves by [Har77, Proposition III.9.2]. Flatness in turn is characterized by the vanishing of $\operatorname{Tor}_{i}^{\mathcal{O}_{B(x)}}\left(\tilde{\mathcal{M}}(x), \kappa_{\beta(x)}\right)$ for all $i>0$ Eis95, Theorem 6.8], and for $\beta \in B_{\mathbb{C}}$, $\operatorname{Tor}_{i}^{\mathcal{O}_{B(x)}}\left(\tilde{\mathcal{M}}(x), \kappa_{\beta(x)}\right) \cong \operatorname{Tor}_{i}^{\mathcal{O}_{B}}\left(\tilde{\mathcal{M}}(x), \kappa_{\beta}\right)$.

Remark 3.2. The result of Theorem 3.1 is false if $B$ is not reduced. Consider for example $B=\operatorname{Spec}\left(\mathbb{C}[b] /\left\langle b^{2}\right\rangle\right)$, and let $\tilde{\mathcal{M}}$ be the sheaf induced by the module $D[b] /\langle\partial, b\rangle$. Since there is only one point in $B$, there is no possibility of a rank-jump. On the other hand, $\operatorname{Tor}_{i}^{\mathcal{O}_{B}}\left(\tilde{\mathcal{M}}(x), \kappa_{\beta}\right)=D(x) /\langle\partial\rangle$ for all $i$.

Theorem 3.1 has an interpretation via Koszul homology. For notation, suppose $T$ is a commutative ring. For a sequence $\mathbf{y}=y_{1}, \ldots, y_{d}$ of elements in $T$, we write the Koszul complex $K \cdot(\mathbf{y})$ [BH93, Chapter 1.6] with lowered indices decreasing from $d$ to 0 . For any $T$-module $N$ set $K \cdot(\mathbf{y} ; N)=K \cdot(\mathbf{y}) \otimes_{T} N$, and abbreviate the $i^{\text {th }}$ Koszul homology of $N$ as $H_{i}(\mathbf{y} ; N)=H_{i}(K \cdot(\mathbf{y} ; N))$. Call $\mathbf{y}$ a regular sequence in $T$ if $T /\langle\mathbf{y}\rangle$ is a nonzero module and $y_{i}$ is a non-zerodivisor on $T /\left\langle y_{1}, \ldots, y_{i-1}\right\rangle$ for $i=1, \ldots, d$. Koszul homology for regular sequences gives Tor groups Eis95, Exercise 17.10], so we get the following.

Corollary 3.3. Let $\mathcal{M}$ be the global sections of a holonomic family over a reduced affine variety $B$ with finitely generated coordinate ring $\mathbb{C}[B]$. If a regular sequence $\mathbf{y}$ in $\mathbb{C}[B]$ generates the ideal of a $\mathbb{C}$-valued closed point $\beta \in B_{\mathbb{C}}$, then $\beta$ is rank-jumping if and only if the Koszul complex $K .(\mathbf{y} ; \mathcal{M}(x))$ has nonzero homology $H_{i}(\mathbf{y} ; \mathcal{M}(x))$ for some $i>0$.

Note that $K \cdot(\mathbf{y} ; \mathcal{M}(x))$ is obtained either by tensoring $K \cdot(\mathbf{y})$ with $\mathcal{M}(x)$ over $\mathbb{C}[B]$, or by viewing $\mathbf{y}$ as a sequence in $\mathbb{C}[B](x)$ and tensoring over $\mathbb{C}[B](x)$.

We now turn to the coherence criterion. We refer to Bjö79, SST00 for more information about filtrations on $D$-modules and their associated graded objects. On $D$ define the order filtration by taking its $k^{\text {th }}$ level to be the vector space of all expressions $\sum_{\nu} p_{\nu}(x) \partial^{\nu}$ in which the monomials $\partial^{\nu}$ for $\nu \in \mathbb{N}^{n}$ have total degree $|\nu| \leq k$. Note that each filtration level is a finitely generated $\mathbb{C}[x]$-module. The associated graded object is the commutative polynomial ring $\operatorname{gr}(D)=\mathbb{C}[x, \xi]$ in the variables $x=x_{1}, \ldots, x_{n}$ and $\xi=\xi_{1}, \ldots, \xi_{n}$.

The order filtration extends to any free module $D^{r}$ with a fixed basis, and to left submodules $K \subseteq D^{r}$, by letting the $k^{\text {th }}$ level be spanned by elements whose coefficients in the given basis have total degree at most $k$ in $\partial$. Given a presentation $N=D^{r} / K$, the left module $N$ has an induced filtration with associated graded module $\operatorname{gr}(N)=\operatorname{gr}\left(D^{r}\right) / \operatorname{gr}(K)$. This naturally graded $\operatorname{gr}(D)$-module depends on the presentation.

Note that since $\mathbb{C}(x)$ is flat over $\mathbb{C}[x]$, the module $\operatorname{gr}(N)(x)$ is isomorphic to $\operatorname{gr}(N(x))$ if we extend the filtration above in the obvious way to $N(x)=\left(D^{r} / K\right)$ $\otimes_{\mathbb{C}[x]} \mathbb{C}(x)$. It is a fundamental fact of $D$-module theory, going back to Kashiwara, that for a holonomic $D$-module $N$, the number $\operatorname{dim}_{\mathbb{C}(x)}(\operatorname{gr}(N)(x))$ is independent of the presentation of $N$ and equals the holonomic rank of $N$ [SST00, Theorem 1.4.19 and Definition 1.4.8].

Our primary use of order filtrations will be on families of $D$-modules over an affine scheme $B$ - that is, on left modules over the ring $D[B]=D \otimes_{\mathbb{C}} \mathbb{C}[B]$. The order 
filtration generalizes naturally to a filtration on $D[B]$ with the property that each level is a finitely generated $\mathbb{C}[B][x]$-module. The associated graded ring of $D[B]$ is the polynomial ring $\mathbb{C}[B][x, \xi]$ in $2 n$ variables over the coordinate ring $\mathbb{C}[B]$. A choice of presentation for a $D[B]$-module $\mathcal{M}$ determines an associated graded $\mathbb{C}[B][x, \xi]$-module $\operatorname{gr}(\mathcal{M})$. The formation of such associated graded structures commutes with the tensor product with the flat $\mathbb{C}[x]$-module $\mathbb{C}(x)$.

Proposition 3.4. For an affine scheme $B$ and a finitely generated $D[B]$-module $\mathcal{M}$, the module $\mathcal{M}(x)$ is finitely generated over $\mathbb{C}[B](x)$ provided that $(\operatorname{gr}(\mathcal{M}))(x)$ is.

Proof. As $B$ is affine, the modules $\mathcal{M}, \mathcal{M}(x)$ and $\operatorname{gr}(\mathcal{M}(x))$ are generated by global sections. Any lift of a set of generators for the $\mathbb{C}[B](x)$-module $(\operatorname{gr} \mathcal{M})(x)$ is a set of generators for $\mathcal{M}(x)$, because the order filtration of $\mathcal{M}(x)$ does not descend infinitely.

We will apply the above result when all fibers $\mathcal{M}_{\beta}$ for $\beta \in B_{\mathbb{C}}$ are holonomic $D$ modules to conclude that $\mathcal{M}$ constitutes (the global sections of) a holonomic family over $B$. It should be pointed out that the finiteness condition on $\mathcal{M}$ is necessary: even if each fiber $\mathcal{M}_{\beta}$ is holonomic, the fiber $(\operatorname{gr} \mathcal{M})(x)_{\beta}$ of the associated graded module $(\operatorname{gr} \mathcal{M})(x)$ over $\beta \in B_{\mathbb{C}}$ is a $\mathbb{C}(x)$-vector space whose dimension need not be equal to $\operatorname{rank}\left(\mathcal{M}_{\beta}\right)$.

Example 3.5. Continue with $\mathcal{M}=D[b] /\langle b x \partial-1\rangle$ as in Remark 2.7. Let $\mathcal{M}(x)_{0} \subseteq$ $\mathcal{M}(x)_{1} \subseteq \cdots$ denote the order filtration of $\mathcal{M}(x)$. Then $\mathcal{M}(x)_{1}=\mathcal{M}(x)$ locally near every parameter $\beta \in \mathbb{C}$ except for $\beta=0$. When $\beta=0$, then by contrast, $\mathcal{M}(x)_{k} / \mathcal{M}(x)_{k-1}$ is minimally generated by $\xi^{k}$, even though $\operatorname{rank}\left(\mathcal{M}_{0}\right)=0$. In fact, $(\operatorname{gr} \mathcal{M})(x)$ is the direct sum of the rank 1 free module $\mathbb{C}[b](x)$ with $\bigoplus_{k \geq 1} \mathbb{C}[b](x) /\langle b\rangle$.

\section{Euler-Koszul homology of toric MODUles}

In this section we introduce generalized $A$-hypergeometric systems in the sense indicated in the introduction. After reviewing some basic facts of GKZ hypergeometric systems, we provide foundations for Euler-Koszul homology of what we call toric $\mathbb{Z}^{d}$-graded modules.

For the rest of this paper, fix a $d \times n$ integer matrix $A=\left(a_{i j}\right)$ of rank $d$. We emphasize that we do not assume that the columns $a_{1}, \ldots, a_{n}$ of $A$ lie in an affine hyperplane. However, we do assume that $A$ is pointed, meaning that $a_{1}, \ldots, a_{n}$ lie in a single open half-space of $\mathbb{R}^{d}$. This guarantees that the semigroup

$$
\mathbb{N} A=\left\{\sum_{i=1}^{n} \gamma_{i} a_{i} \mid \gamma_{1}, \ldots, \gamma_{n} \in \mathbb{N}\right\}
$$

has no units. (Pointedness will come into play in the proof of Theorem 6.6, where it is used to ensure that local duality holds.) The semigroup ring associated to the $d \times n$ matrix $A$ is $S_{A}=\mathbb{C}[\mathbb{N} A] \cong R / I_{A}$, where $R=\mathbb{C}\left[\partial_{1}, \ldots, \partial_{n}\right]$ and

$$
I_{A}=\left\langle\partial^{\mu}-\partial^{\nu} \mid \mu, \nu \in \mathbb{Z}^{n}, A \cdot \mu=A \cdot \nu\right\rangle
$$

is the toric ideal of $A$. Notice that $S_{A}$ and $R$ are naturally graded by $\mathbb{Z}^{d}$ if we define $\operatorname{deg}\left(\partial_{j}\right)=-a_{j}$, the negative of the $j^{\text {th }}$ column of $A$.

Our choice of signs in the $\mathbb{Z}^{d}$-grading of $R$ is compatible with a $\mathbb{Z}^{d}$-grading on the Weyl algebra $D$ in which $\operatorname{deg}\left(x_{j}\right)=a_{j}$ and $\operatorname{deg}\left(\partial_{j}\right)=-a_{j}$. Under this 
$\mathbb{Z}^{d}$-grading, the $i^{\text {th }}$ Euler operator $E_{i}=\sum_{j=1}^{n} a_{i j} x_{j} \partial_{j} \in D$ is homogeneous of degree 0 for $i=1, \ldots, d$. Given a vector $\beta \in \mathbb{C}^{d}$, we write $E-\beta$ for the sequence $E_{1}-\beta_{1}, \ldots, E_{d}-\beta_{d}$.

Definition 4.1. The A-hypergeometric GKZ system with parameter $\beta$ is the left ideal

$$
H_{A}(\beta)=D \cdot\left\langle I_{A}, E-\beta\right\rangle
$$

in the Weyl algebra $D$. The $A$-hypergeometric $D$-module with parameter $\beta$ is

$$
\mathcal{M}_{\beta}^{A}=D / H_{A}(\beta) .
$$

Results of [GKZ89, Ado94, Hot98, SST00] imply that the module $\mathcal{M}_{\beta}^{A}$ is holonomic of nonzero rank. $A$-hypergeometric systems constitute an important class of $D$-modules, playing a role similar to that of toric varieties in algebraic geometry, since they possess enough combinatorial underpinning to make calculations feasible, and enough diversity of behavior to make them interesting as a test class for conjectures and computer experimentation.

If $y \in N_{\alpha}$ is homogeneous of degree $\alpha$ in a $\mathbb{Z}^{d}$-graded $D$-module $N$, write $\operatorname{deg}_{i}(y)$ $=\alpha_{i}$. In particular, for any homogeneous element $P \in D$,

$$
E_{i} P-P E_{i}=\operatorname{deg}_{i}(P) P .
$$

The displayed equation shows that if $N$ is any $\mathbb{Z}^{d}$-graded left $D$-module, then the map $E_{i}-\beta_{i}: N \rightarrow N$ sending the homogeneous element $y \in N$ to

$$
\left(E_{i}-\beta_{i}\right) \circ y=\left(E_{i}-\beta_{i}-\operatorname{deg}_{i}(y)\right) y
$$

determines a $D$-linear endomorphism of $N$ when extended $\mathbb{C}$-linearly to inhomogeneous elements of $N$. This endomorphism is functorial, in the sense that it commutes with degree 0 homomorphisms $N \rightarrow N^{\prime}$ of $\mathbb{Z}^{d}$-graded left $D$-modules. Moreover, the endomorphisms for the various Euler operators commute: $\left[E_{i}-\beta_{i}, E_{j}-\beta_{j}\right]$ is the zero endomorphism on any $\mathbb{Z}^{d}$-graded left $D$-module for all $i, j=1, \ldots, d$ and all complex numbers $\beta_{i}, \beta_{j}$.

Definition 4.2. Fix $\beta \in \mathbb{C}^{d}$ and a $\mathbb{Z}^{d}$-graded $R$-module $N$. The Euler-Koszul complex $\mathcal{K} \cdot(E-\beta ; N)$ is the Koszul complex of left $D$-modules defined by the sequence $E-\beta$ of commuting endomorphisms on the left $D$-module $D \otimes_{R} N$ concentrated in homological degrees $d$ to 0 . The $i^{\text {th }}$ Euler-Koszul homology of $N$ is $\mathcal{H}_{i}(E-\beta ; N)=H_{i}(\mathcal{K} \cdot(E-\beta ; N))$.

This complex was used before in the special case $N=S_{A}$ in GKZ89, Ado94, Ado99 in order to study the solutions to $H_{A}(\beta)$ for special parameters $\beta$. We use the script ' $\mathcal{K}$ ' for Euler-Koszul complexes instead of the usual ' $K$ ' for ordinary Koszul complexes to emphasize that the maps in $\mathcal{K} .(E-\beta ; N)$ are homomorphisms of $D$-modules. The action of an endomorphism in the sequence $E-\beta$ on a homogeneous element depends on the degree of that element, in contrast to maps in Koszul complexes over commutative graded rings. Nonetheless, Euler-Koszul complexes behave much like ordinary Koszul complexes. To see why, let $\theta_{i}=x_{i} \partial_{i} \in D$. In the $\mathbb{Z}^{d}$-graded commutative subalgebra $\Theta=\mathbb{C}\left[\theta_{1}, \ldots, \theta_{n}\right]$ of $D$ every element has degree $0 \in \mathbb{Z}^{d}$. Consequently, $N_{\alpha}$ is a left $\Theta$-module whenever $N$ is a $\mathbb{Z}^{d}$-graded $D$-module. We hence obtain the following. 
Lemma 4.3. Let $N$ be a $\mathbb{Z}^{d}$-graded $R$-module and $\alpha \in \mathbb{Z}^{d}$. The $\mathbb{Z}^{d}$-graded degree $\alpha$ part $\mathcal{K} .(E-\beta ; N)_{\alpha}$ of the Euler-Koszul complex agrees with the ordinary Koszul complex $K .\left(E-\beta-\alpha ;\left(D \otimes_{R} N\right)_{\alpha}\right)$ constructed over the polynomial subalgebra $\Theta \subseteq D$. That is,

$$
\mathcal{K} \cdot(E-\beta ; N)=\bigoplus_{\alpha \in \mathbb{Z}^{d}} K \cdot\left(E-\beta-\alpha ;\left(D \otimes_{R} N\right)_{\alpha}\right)
$$

the right-hand side being a direct sum of ordinary Koszul complexes of $\Theta$-modules.

Since $D$ is a free $R$-module, the Euler-Koszul complex constitutes an exact functor from the category of $\mathbb{Z}^{d}$-graded $R$-modules with $\mathbb{Z}^{d}$-graded morphisms of degree 0 to the category of bounded complexes of $\mathbb{Z}^{d}$-graded left $D$-modules with $\mathbb{Z}^{d}$-graded morphisms of degree 0 . Consequently, short exact sequences of $\mathbb{Z}^{d}$-graded modules over $R$ give rise to long exact sequences of Euler-Koszul homology.

We now specify a subcategory of the category of $R$-modules that is central to what follows. This subcategory will contain the semigroup ring $S_{A}$ as well as its monomial (that is, $\mathbb{Z}^{d}$-graded) ideals, and it will be closed under taking $\mathbb{Z}^{d}$-graded submodules, quotient modules, and extensions. In the following section we then study the Euler-Koszul homology of objects in this category and establish strong (non)vanishing conditions.

Notation 4.4. By a face of $A$ we mean a set $F$ of columns of $A$ minimizing some linear functional on $\mathbb{N} A$. We interpret $F$ as a submatrix of $A$. The $d i$ mension $\operatorname{dim}(F)$ of a face $F$ equals the rank of the subgroup $\mathbb{Z} F \subseteq \mathbb{Z}^{d}$ it generates. Let $S_{F}$ be the semigroup ring generated by $F$. Thus $S_{F} \cong R / I_{A}^{F}$, where $I_{A}^{F}=R \cdot I_{F}+\left\langle\partial_{j} \mid a_{j} \notin F\right\rangle$ is the prime ideal obtained by starting with $I_{F}$ (defined as the kernel of the surjection $\mathbb{C}\left[\partial_{j} \mid a_{j} \in F\right] \rightarrow S_{F}$ ) and adding the variables corresponding to the columns of $A$ outside of $F$.

Definition 4.5. A $\mathbb{Z}^{d}$-graded $R$-module $M$ is toric if it has a toric filtration

$$
0=M_{0} \subset M_{1} \subset \cdots \subset M_{\ell-1} \subset M_{\ell}=M,
$$

meaning that $M_{k} / M_{k-1}$ is, for each $k$, a $\mathbb{Z}^{d}$-graded translate of $S_{F_{k}}$ for some face $F_{k}$ of $\mathbb{N} A$. We say that $M$ has toric length $\ell$ if the minimal length of a toric filtration for $M$ is $\ell$.

Remark 4.6. Most toric modules have many different toric filtrations, and usually more than one of these has minimal length. A toric module of toric length 1 is simply a $\mathbb{Z}^{d}$-graded translate $S_{F}(-\alpha)$ of $S_{F}$ generated in degree $\alpha \in \mathbb{Z}^{d}$, for some face $F$ of $A$.

Example 4.7. If $N$ is a finitely generated $\mathbb{Z}^{d}$-graded $S_{F}$-module for some face $F$ of $A$, then $N$ is toric. To see this, argue by induction on $\operatorname{dim}(N)$, the Artinian case $\operatorname{dim}(N)=0$ being trivial. If $\operatorname{dim}(N)>0$, then $N$ has a submodule of the form $S_{F^{\prime}}(-\alpha)$ with $F^{\prime} \subseteq F$ being some face of $A$ of $\operatorname{dimension} \operatorname{dim}(N)$. If we replace $N$ by $N / S_{F^{\prime}}(\alpha)$, then a finite number of such steps will reduce the dimension of $N$ by at least one.

Example 4.7 implies that $\mathbb{Z}^{d}$-graded submodules, quotients, and extensions of toric modules are toric, since they have a composition chain by shifts of modules of the form $S_{F}$.

If $F$ is a face of $A$, then we write $E_{i}^{F}$ for the Euler operator obtained by erasing all terms $a_{i j} x_{j} \partial_{j}$ from $E_{i}$ such that $a_{j} \notin F$. We will now consider the actions 
of Euler endomorphisms $E_{i}-\beta_{i}$ on $D \otimes_{R} M$ for an $S_{F}$-module $M$. To describe such actions, let $D_{F}$ and $D_{\bar{F}}$ be the Weyl algebras on the variables $\left\{x_{F}, \partial_{F}\right\}$ and $\left\{x_{\bar{F}}, \partial_{\bar{F}}\right\}$ corresponding to the columns $a_{j} \in F$ and $a_{j} \notin F$, respectively. Then for each $S_{F}$-module $M$ we have

$$
D \otimes_{R} M \cong \mathbb{C}\left[x_{\bar{F}}\right] \otimes_{\mathbb{C}}\left(D_{F} \otimes_{\mathbb{C}\left[\partial_{F}\right]} M\right)
$$

as left modules over $D=D_{\bar{F}} \otimes_{\mathbb{C}} D_{F}$, identifying the element $x_{F}^{\mu} x_{\bar{F}}^{\bar{\mu}} \partial_{F}^{\nu} \partial \frac{\bar{\nu}}{F} \otimes m$ with $x_{\bar{F}}^{\bar{\mu}} \otimes\left(x_{F}^{\mu} \partial_{F}^{\nu} \otimes \partial \overline{\bar{\nu}} m\right)$, where of course both sides are zero if $|\bar{\nu}|>0$.

Suppose that $M$ is a $\mathbb{Z}^{d}$-graded $S_{F}$-module and interpret it as a $\mathbb{C}\left[\partial_{F}\right]$-module (rather than as a module over a quotient of $R$ ). Note that $E_{i}^{F}$ induces an Euler endomorphism on $D_{F} \otimes_{\mathbb{C}\left[\partial_{F}\right]} M$ by setting $E_{i}^{F} \circ(P \otimes m)=\left(\left(\sum_{j \in F} a_{i j} x_{j} \partial_{j}\right)-\right.$ $\left.\operatorname{deg}_{i}(P \otimes m)\right) P \otimes m$ for all homogeneous elements $P \in D_{F}$ and $m \in M$.

Lemma 4.8. If $M$ is a finitely generated $\mathbb{Z}^{d}$-graded $S_{F}$-module, then under the isomorphism (it) above, the action of the endomorphism $E_{i}-\beta_{i}$ on $D \otimes_{R} M$ coincides with the action of the endomorphism $E_{i}^{F}-\beta_{i}$ on the right-hand factor of $\mathbb{C}\left[x_{\bar{F}}\right] \otimes_{\mathbb{C}}$ $\left(D_{F} \otimes_{\mathbb{C}\left[\partial_{F}\right]} M\right)$ :

$$
\left(E_{i}-\beta_{i}\right) \circ\left(x_{F}^{\mu} x_{\frac{\bar{\mu}}{F}} \partial_{F}^{\nu} \partial \frac{\bar{\nu}}{F} \otimes m\right)=x_{\frac{\bar{\mu}}{F}}^{\bar{F}} \otimes\left(E_{i}^{F}-\beta_{i}\right) \circ\left(x_{F}^{\mu} \partial_{F}^{\nu} \otimes \partial \frac{\bar{\nu}}{F} m\right) .
$$

Proof. Let $P=x_{F}^{\mu} x_{\bar{F}}^{\bar{\mu}} \partial_{F}^{\nu} \partial \frac{\bar{\nu}}{F}$ and $P^{\prime}=x_{F}^{\mu} \partial_{F}^{\nu} \partial \frac{\bar{\nu}}{F}$. Note that $\left[E_{i}^{F}, x_{\bar{F}}^{\bar{\mu}}\right]=0$ and therefore $\left[E_{i}-E_{i}^{F}, x_{\bar{F}}^{\bar{\mu}}\right]=\operatorname{deg}_{i}\left(x_{\bar{F}}^{\bar{\mu}}\right) x_{\bar{F}}^{\bar{\mu}}$. We then compute

$$
\begin{aligned}
\left(E_{i}-\beta_{i}\right) \circ(P \otimes m)= & \left(E_{i}-\beta_{i}-\operatorname{deg}_{i}(P \otimes m)\right) \cdot P \otimes m \\
= & \left(\left(E_{i}-E_{i}^{F}\right)-\operatorname{deg}_{i}\left(x_{\bar{F}}^{\bar{\mu}}\right)\right) P \otimes m \\
& +\left(E_{i}^{F}-\beta_{i}-\operatorname{deg}_{i}\left(P^{\prime} \otimes m\right)\right) P \otimes m \\
= & 0+x_{\bar{F}}^{\bar{\mu}}\left(E_{i}^{F}-\beta_{i}-\operatorname{deg}_{i}\left(P^{\prime} \otimes m\right)\right) P^{\prime} \otimes m,
\end{aligned}
$$

which is identified with $x_{\bar{F}}^{\bar{\mu}} \otimes\left(E_{i}^{F}-\beta_{i}-\operatorname{deg}_{i}\left(P^{\prime} \otimes m\right)\right) P^{\prime} \otimes m$.

In analogy with the construction of the $A$-hypergeometric system $\mathcal{M}_{\beta}^{A}$ from $A$, the faces of $A$ give rise to holonomic $D$-modules, but sometimes these modules can be zero.

Lemma 4.9. Fix a vector $\beta \in \mathbb{C}^{d}$ and a face $F$ of $A$. The following are equivalent for the quotient $\mathcal{M}_{\beta}^{F}:=D /\left\langle I_{A}^{F}, E-\beta\right\rangle$.

1. The module $\mathcal{M}_{\beta}^{F}$ is nonzero.

2. $\mathcal{M}_{\beta}^{F}$ is a holonomic D-module of nonzero rank.

3. The parameter $\beta$ (or, equivalently, $-\beta$ ) lies in the span (over $\mathbb{C}$ ) of the columns of $F$.

Proof. If $\beta$ lies outside the column-span of $F$, then the $\mathbb{C}$-linear span of $E-\beta$ and the products $\left\{x_{j} \partial_{j} \mid a_{j} \notin F\right\} \subset D \cdot I_{A}^{F}$ contains a nonzero scalar, so $\mathcal{M}_{\beta}^{F}$ is zero, as is its rank.

Suppose now that $\beta$ lies in the column-span of $F$. Let $F^{\prime}$ be a submatrix of $F$ composed of $\operatorname{dim}(F)$ many linearly independent rows of $F$, and let $\beta^{\prime}$ be the part of $\beta$ in the corresponding rows. Then the hypergeometric system $\mathcal{M}_{\beta^{\prime}}^{F^{\prime}}:=$ $D_{F} /\left\langle I_{F}, E^{F}-\beta\right\rangle$ associated to the matrix $F^{\prime}$ and the parameter vector $\beta^{\prime}$ is a holonomic $D_{F}$-module of nonzero rank, by GKZ89, Ado94]. Since $\mathbb{C}\left[x_{\bar{F}}\right] \otimes_{\mathbb{C}} \mathcal{M}_{\beta^{\prime}}^{F^{\prime}} \cong$ 
$\mathcal{M}_{\beta}^{F}$ by Lemma 4.8, $\mathcal{M}_{\beta}^{F}$ is holonomic over $D=D_{\bar{F}} \otimes_{\mathbb{C}} D_{F}$ and has nonzero rank.

We close this section by defining the main object of study of the remainder of the paper.

Definition 4.10. Let $M$ be a toric $R$-module and $\beta \in \mathbb{C}^{d}$. The generalized hypergeometric system associated to $M$ and $\beta$ is the zeroth Euler-Koszul homology $\mathcal{H}_{0}(E-\beta ; M)$.

If $M=S_{A}$, then $\mathcal{H}_{0}(E-\beta ; M)$ is the holonomic GKZ-module $\mathcal{M}_{\beta}^{A}$ from Definition 4.1 .

\section{Rigidity and holonomicity of Euler-Koszul homology}

We begin our treatment of generalized hypergeometric systems by showing that they are holonomic, as are all of the higher Euler-Koszul homology modules of toric modules.

Proposition 5.1. The generalized hypergeometric system $\mathcal{H}_{0}(E-\beta ; M)$ is holonomic for every toric module $M$ and every parameter $\beta \in \mathbb{C}^{d}$. Consequently, $\mathcal{H}_{i}(E-\beta ; M)$ is holonomic for all $i>0$ as well.

Proof. Consider first the case of $\mathcal{H}_{0}(E-\beta ; M)$. The proof is by induction on the toric length $\ell$ of $M$. When $M=S_{F}$ for some face $F$, use Lemma 4.9 The general $\ell=1$ case follows because $\mathcal{H}_{0}\left(E-\beta ; S_{F}(\alpha)\right) \cong \mathcal{H}_{0}\left(E-\beta+\alpha ; S_{F}\right)(\alpha)$. For $\ell>1$, the Euler-Koszul functor applied to a toric short exact sequence $M_{1} \hookrightarrow M \rightarrow M / M_{1}$ induces a sequence

$$
\mathcal{H}_{0}\left(E-\beta ; M_{1}\right) \rightarrow \mathcal{H}_{0}(E-\beta ; M) \rightarrow \mathcal{H}_{0}\left(E-\beta ; M / M_{1}\right)
$$

that is exact and has holonomic modules at both ends by induction.

Now consider $i>0$. Let $m \in \operatorname{ker}\left(\mathcal{K}_{i}(E-\beta ; M) \rightarrow \mathcal{K}_{i-1}(E-\beta ; M)\right)$, so its coset $\bar{m}$ is an element of $\mathcal{H}_{i}(E-\beta ; M)$. Since the Euler-Koszul complex is $\mathbb{Z}^{d}$-graded, we may assume that $m$ is homogeneous of degree $\alpha \in \mathbb{Z}^{d}$. Note that $\mathcal{H}_{i}(E-\beta ; M)$ is generated by finitely many such $\bar{m}$, since $\mathcal{K}_{i}(E-\beta ; M)$ is a direct sum of $\left(\begin{array}{l}d \\ i\end{array}\right)$ copies of $\left(D \otimes_{R} M\right)$, which is a Noetherian $D$-module. It is hence sufficient to prove that $D \cdot \bar{m}$ is holonomic.

Consider the Koszul complex $K \cdot\left(E-\beta-\alpha,\left(D \otimes_{R} M\right)_{\alpha}\right)$ on the (left) $\Theta$-module $\left(D \otimes_{R} M\right)_{\alpha}$. Within this complex, $m$ descends to an element $\bar{m}$ of the $i^{\text {th }}$ homology module $H_{i}\left(E-\beta-\alpha,\left(D \otimes_{R} M\right)_{\alpha}\right)$. We remark that the two usages of $\bar{m}$ agree in the sense of the isomorphism in Lemma 4.3. Since $\Theta$ is commutative, left multiplication by $E_{j}-\beta_{j}-\alpha_{j}$ annihilates $\bar{m}$ by [BH93, Proposition 1.6.5]. On the other hand, $m$ lies in the direct sum of $\left(\begin{array}{l}d \\ i\end{array}\right)$ copies of $\left(D \otimes_{R} M\right)$. Since $I_{A} \cdot M=0$, there is some integer $k \in \mathbb{N}$ such that $\left(I_{A}\right)^{k} m=0$. It follows that the $D$-module $D \cdot \bar{m}$ is a $\mathbb{Z}^{d}$-graded quotient of $D /\left\langle E-\beta-\alpha,\left(I_{A}\right)^{k}\right\rangle$. In particular, $D \cdot \bar{m}$ has a finite composition series such that each composition factor is a quotient of $D /\left\langle E-\beta-\alpha, I_{A}\right\rangle \cong \mathcal{H}_{0}\left(E-\beta-\alpha ; S_{A}\right)$ and hence is holonomic by the first sentence of the proposition.

Definition 5.2. Let $N$ be any $\mathbb{Z}^{d}$-graded $R$-module. A vector $\alpha \in \mathbb{Z}^{d}$ is a true degree of $N$, written $\alpha \in \operatorname{tdeg}(N)$, if the graded piece $N_{\alpha}$ is nonzero. A vector $\alpha \in \mathbb{C}^{d}$ is a quasi-degree of $N$, written $\alpha \in \operatorname{qdeg}(N)$, if $\alpha$ lies in the complex Zariski closure qdeg $(N)$ of the true degrees of $N$ via the natural embedding $\mathbb{Z}^{d} \hookrightarrow \mathbb{C}^{d}$. 
We now prove a rigidity property of the Euler-Koszul complex.

Proposition 5.3. For a toric $R$-module $M$ and $\beta \in \mathbb{C}^{d}$ the following are equivalent.

1. $\mathcal{H}_{0}(E-\beta ; M)$ has holonomic rank 0 .

2. $\mathcal{H}_{0}(E-\beta ; M)=0$.

3. $\mathcal{H}_{i}(E-\beta ; M)=0$ for all $i \geq 0$.

4. $-\beta \notin \operatorname{qdeg}(M)$.

Proof. $2 \Leftrightarrow 3$ : The failure of the last condition in Lemma 4.9 is equivalent to the $\mathbb{C}$-span of $E-\beta$ containing an endomorphism whose action on $D / D \cdot I_{A}^{F}$ is multiplication by a nonzero scalar. This observation, together with the isomorphism $\mathcal{H}_{0}\left(E-\beta ; S_{F}(\alpha)\right) \cong \mathcal{H}_{0}\left(E-\beta+\alpha ; S_{F}\right)(\alpha)$, shows that $2 \Leftrightarrow 3$ when $M$ has toric length 1 . Now suppose that $M$ has toric length $\ell>1$, and assume the result for modules of smaller toric length. For any toric filtration of $M$, the long exact sequence of Euler-Koszul homology for $M_{1} \hookrightarrow M \rightarrow M / M_{1}$ shows that $\mathcal{H}_{0}(E-\beta ; M)$ surjects onto $\mathcal{H}_{0}\left(E-\beta ; M / M_{1}\right)$. Assuming that the former is zero, so is the latter. By induction, we find that $\mathcal{H}_{i}\left(E-\beta ; M / M_{1}\right)$ vanishes for all $i \geq 0$, and hence that $\mathcal{H}_{i}(E-\beta ; M) \cong \mathcal{H}_{i}\left(E-\beta ; M_{1}\right)$ for all $i \geq 0$. The result now holds for $M$ because it holds by induction for $M_{1}$.

$1 \Leftrightarrow 2$ : We need that $\mathcal{H}_{0}(E-\beta ; M) \neq 0$ implies that its rank is nonzero. Again use induction on the toric length $\ell$. For $\ell=1$ this is Lemma 4.9 plus the isomorphism $\mathcal{H}_{0}\left(E-\beta ; S_{F}(\alpha)\right) \cong \mathcal{H}_{0}\left(E-\beta+\alpha ; S_{F}\right)(\alpha)$, so assume $\ell>1$. Again make the observation that $\mathcal{H}_{0}(E-\beta ; M)$ surjects onto $\mathcal{H}_{0}\left(E-\beta ; M / M_{1}\right)$. If this latter module is nonzero, then it has nonzero rank by induction, so $\mathcal{H}_{0}(E-\beta ; M)$ has nonzero rank, too. Hence we assume that $\mathcal{H}_{0}\left(E-\beta ; M / M_{1}\right)=0$. Now, using the equivalence $2 \Leftrightarrow 3$, we find that $\mathcal{H}_{0}(E-\beta ; M) \cong \mathcal{H}_{0}\left(E-\beta ; M_{1}\right)$, so again we are done by induction.

$2 \Leftrightarrow 4$ : For the semigroup ring $S_{F}$ of a face, $\mathcal{H}_{0}\left(E-\beta ; S_{F}\right)=\mathcal{M}_{\beta}^{F}$ is the $D$ module from Lemma 4.9. Therefore $2 \Leftrightarrow 4$ for $M=S_{F}$. For $\mathbb{Z}^{d}$-graded translates of $S_{F}$,

$$
\left[\mathcal{H}_{0}\left(E-\beta ; S_{F}\right) \neq 0\right] \Leftrightarrow\left[-\beta \in \operatorname{qdeg}\left(S_{F}\right)\right] \Leftrightarrow\left[-\beta-\alpha \in \operatorname{qdeg}\left(S_{F}(\alpha)\right)\right]
$$

for $\alpha \in \mathbb{Z}^{d}$, but also $\left[\mathcal{H}_{0}\left(E-\beta ; S_{F}\right) \neq 0\right] \Leftrightarrow\left[\mathcal{H}_{0}\left(E-\beta-\alpha ; S_{F}(\alpha)\right) \neq 0\right]$ by definition of the endomorphisms $E_{i}-\beta_{i}$. This proves $2 \Leftrightarrow 4$ when $M$ has toric length 1 . To treat modules of toric length $\ell>1$, consider a toric sequence $M_{1} \hookrightarrow M \rightarrow M / M_{1}$. Since $q \operatorname{deg}(M)=\operatorname{qdeg}\left(M_{1}\right) \cup \operatorname{qdeg}\left(M / M_{1}\right)$, we find that $-\beta \in \operatorname{qdeg}(M)$ if and only if

$$
\left[-\beta \in \operatorname{qdeg}\left(M_{1}\right)\right] \quad \text { or } \quad\left[-\beta \in \operatorname{qdeg}\left(M / M_{1}\right)\right] .
$$

This condition is equivalent by induction on $\ell$ to

$$
\left[\mathcal{H}_{0}\left(E-\beta ; M_{1}\right) \neq 0\right] \quad \text { or } \quad\left[\mathcal{H}_{0}\left(E-\beta ; M / M_{1}\right) \neq 0\right] .
$$

Now $\mathcal{H}_{0}(E-\beta ; M)$ always surjects onto $\mathcal{H}_{0}\left(E-\beta ; M / M_{1}\right)$, and if $\mathcal{H}_{0}\left(E-\beta ; M / M_{1}\right)$ vanishes, then by the equivalence $2 \Leftrightarrow 3, \mathcal{H}_{0}\left(E-\beta ; M_{1}\right) \cong \mathcal{H}_{0}(E-\beta ; M)$. Therefore, this last displayed condition is equivalent to $\mathcal{H}_{0}(E-\beta ; M) \neq 0$.

\section{Euler-Koszul homology detects local COHOMOlOGY}

In this section we describe the set of parameters $\beta \in \mathbb{C}^{d}$ for which the EulerKoszul complex $\mathcal{K} .(E-\beta ; N)$ has nonzero higher homology. Namely, we identify this set with the quasi-degrees of the local cohomology of the toric module $N$. 
Our proof of this result in Theorem 6.6 uses a spectral sequence, constructed in Theorem 6.3. that arises from the holonomic duality functor. For some background on holonomic duality we refer to Bjö79.

For us, duality $\mathbb{D}$ for (complexes of) $D$-modules is the combination of the derived functor $\mathbb{R H o m}_{D}\left({ }_{-}, D\right)$ of homomorphisms to $D$ followed by the involution $\tau$ taking $x^{\mu} \partial^{\nu}$ to

$$
\tau\left(x^{\mu} \partial^{\nu}\right)=(-\partial)^{\nu} x^{\mu} .
$$

Hence, in order to compute the dual $\mathbb{D}(N)$ of the module $N$ placed in homological degree zero, let $F$. be a $D$-free resolution of $N$ and apply $\tau$ to $\operatorname{Hom}_{D}(F, D)$. Holonomicity of $N$ is equivalent to $\mathbb{D}(N)$ being exact in all cohomological degrees but $n$. If $N$ is a module in homological degree $k$, then the cohomology $H^{i} \mathbb{D}(N)$ is nonzero only for $i=k+n$. Therefore $\mathbb{D}$ constitutes an exact functor from the category of complexes of $D$-modules with holonomic homology to itself, and $\mathbb{D}(\mathbb{D}(N))=N$ for all $N$.

The rank of a holonomic module $N$ equals that of its dual: $\operatorname{rank}(N)=\operatorname{rank}(\mathbb{D}(N))$. To be more precise, locally near a nonsingular point of the complex analytic manifold $\mathbb{C}_{a n}^{n}, N$ is a connection: $N_{a n}=D_{a n}{ }^{r} /\left\langle\partial_{j}-C_{j} \mid j=1, \ldots, n\right\rangle$ where $C_{j} \in \mathcal{O}_{a n}{ }^{r, r}$ are $r \times r$ matrices of holomorphic functions and one has vanishing commutators $\left[\partial_{i}-C_{i}, \partial_{j}-C_{j}\right]$. Hence the right Koszul complex $K \cdot\left(\partial-C, D_{a n}\right)$ on the operators $\partial_{i}-C_{i}$ is a free resolution of $N_{a n}$ and the holonomic dual is computed from this Koszul complex. In particular, $\mathbb{D}\left(N_{a n}\right)=D_{a n}{ }^{r} /\left\langle\partial_{j}+C_{j} \mid j=1, \ldots, n\right\rangle$ and so $\operatorname{rank}(N)=\operatorname{rank}(\mathbb{D}(N))=r$.

The automorphism $x \mapsto-x$ on $\mathbb{C}^{n}$ induces, via $\partial \mapsto-\partial$, auto-equivalences $N \mapsto$ $N^{-}$on the categories of $R$-modules and of $D$-modules (but not on the category of $\mathbb{Z}^{d}$-graded $S_{A^{-}}$-modules, since $I_{A}$ is not preserved under (_ $)^{-}$unless it is projective). The formation of Euler-Koszul complexes is equivariant under this sign change since $E_{i}-\beta_{i}=\left(E_{i}-\beta_{i}\right)^{-}$. Moreover, for $\mathbb{Z}^{d}$-graded $R$-modules $N$ we have $D \otimes_{R} N \cong$ $\tau\left(N \otimes_{R} D\right)^{-}$as $D$-modules, where the tensor products exploit the two different $R$-structures on $D$.

The ordinary Koszul complex $K \cdot(\mathbf{y} ; T)$ on a sequence $\mathbf{y}=y_{1}, \ldots, y_{d}$ in a commutative $\operatorname{ring} T$ is isomorphic to its dual $K^{\bullet}(\mathbf{y} ; T)=\operatorname{Hom}_{T}(K \cdot(\mathbf{y} ; T), T)$. In fact, $K \cdot(\mathbf{y} ; T)$ equals $K^{\bullet}(\mathbf{y} ; T)$ after replacing each lowered homological index $i$ by the raised cohomological index $d-i$ and a suitable sign change in the differentials. Administering this sign- and index-change to $\mathcal{K} \cdot(E-\beta ; N)$ in Definition 4.2 yields $\mathcal{K}^{\bullet}(E-\beta ; N)$, whose cohomology we call the Euler-Koszul cohomology of $N$; we have $\mathcal{H}^{i}(E-\beta ; N) \cong \mathcal{H}_{d-i}(E-\beta ; N)$.

We shall apply $\mathcal{K} .\left(E-\beta ;_{-}\right)$and $\mathcal{K}^{\bullet}\left(E-\beta ;_{-}\right)$to $\mathbb{Z}^{d}$-graded complexes of $R$ modules. Our conventions for indexing the resulting double complexes are set up as follows, so that all homological and cohomological indices are positive. If $\mathcal{F}_{.}: \mathcal{F}_{0} \leftarrow \mathcal{F}_{1} \leftarrow \cdots \leftarrow \mathcal{F}_{n}$ is a complex of $R$-modules with decreasing lowered indices, then we write the differentials of the double complex $\mathcal{K} .(E-\beta ; \mathcal{F}$. $)$ pointing downward (the $\mathcal{K}$. direction) and to the left (the $\mathcal{F}$. direction). On the other hand, if $\mathcal{F}^{\cdot}: \mathcal{F}^{0} \rightarrow \mathcal{F}^{1} \rightarrow \cdots \rightarrow \mathcal{F}^{n}$ has increasing raised indices, then we write the double complex $\mathcal{K}^{\bullet}\left(E-\beta ; \mathcal{F}^{\bullet}\right)$ with differentials pointing upward and to the right. Applying $\operatorname{Hom}_{T}(,, T)$ to a complex of free $T$-modules with decreasing lowered indices (with $T=R$ or $T=D$ ) yields a complex with increasing raised indices.

Our theory of toric modules applies to the modules $\operatorname{Ext}_{R}^{i}(M, R)$ whenever $M$ is toric. 
Lemma 6.1. If $M$ is a toric $R$-module, then $\operatorname{Ext}_{R}^{i}(M, R)$ is toric for all $i$.

Proof. The module $M$ has a composition chain by $\mathbb{Z}^{d}$-graded $S_{A}$-modules. If $M$ is of toric length 1 , then the finitely generated $S_{A}$-module $\operatorname{Ext}_{R}^{i}(M, R)$ is toric by Example 4.7.

In the general case, argue by induction on the toric length of $M$. The $\mathbb{Z}^{d}$ graded long exact sequence of $\operatorname{Ext}_{R}(-, R)$ arising from $M_{1} \hookrightarrow M \rightarrow M / M_{1}$ places $\operatorname{Ext}_{R}^{i}(M, R)$ between two modules both of which are toric by induction.

Definition 6.2. Let $\varepsilon_{A}$ be the sum $\sum_{j=1}^{n} a_{j}$ of the columns of $A$. Using our convention that $\operatorname{deg}\left(\partial_{j}\right)=-a_{j}$, the canonical module of $R$ is $\omega_{R}=R\left(\varepsilon_{A}\right)$.

Theorem 6.3. If $M$ is a toric $R$-module, then there is a spectral sequence

$$
E_{2}^{p, q}=\mathcal{H}^{q}\left(E+\beta ; \operatorname{Ext}_{R}^{p}\left(M, \omega_{R}\right)\right)\left(-\varepsilon_{A}\right) \Longrightarrow \mathbb{D}\left(\mathcal{H}_{p+q-n}(E-\beta ; M)\right)^{-},
$$

where we place $\mathcal{H}_{p+q-n}(E-\beta ; M)$ in homological degree $p+q-n$.

Proof. Let $\mathcal{F}$. be a minimal $\mathbb{Z}^{d}$-graded $R$-free resolution of $M$. Consider the double complex $\tau \operatorname{Hom}_{D}(\mathcal{K} \cdot(E-\beta ; \mathcal{F} \cdot), D)^{-}$. With $\mathcal{F}^{\cdot}=\operatorname{Hom}_{R}(\mathcal{F} \cdot, R)$, we claim that

$$
\tau \operatorname{Hom}_{D}(\mathcal{K} \cdot(E-\beta ; \mathcal{F} \cdot), D)^{-} \cong \mathcal{K}^{\bullet}\left(-E-\beta-\varepsilon_{A} ; \mathcal{F}^{*}\right) .
$$

To see why, begin by noting that $\tau\left({ }_{-}\right)^{-}$is an isomorphism when $D$-modules are regarded as $R$-modules, but identifies (for example) the left $D$-module $D \otimes_{R} S_{A}$ with the right $D$-module $S_{A} \otimes_{R} D$. Hence each row of $\tau \operatorname{Hom}_{D}(\mathcal{K} \cdot(E-\beta ; \mathcal{F} \cdot), D)^{-}$ is a direct sum of complexes of the form $\tau\left(\mathcal{F}^{*} \otimes_{R} D\right)^{-}$with cohomology $D \otimes_{R}$ $\operatorname{Ext}_{R}^{i}(M, R)$.

On the other hand, note that $\tau\left(E_{i}-\beta_{i}\right)^{-}=-E_{i}-\beta_{i}-\left(\varepsilon_{A}\right)_{i}$. Therefore each column of $\tau \operatorname{Hom}_{D}(\mathcal{K} .(E-\beta ; \mathcal{F} \cdot), D)^{-}$is an Euler-Koszul cocomplex induced by $-E-\beta-\varepsilon_{A}$ on $\tau\left(\mathcal{F}^{i} \otimes_{R} D\right)^{-}$. We consider the spectral sequences associated to this double complex.

Taking first the horizontal and then the vertical cohomology of the double complex, we obtain $\mathcal{H}^{q}\left(-E-\beta-\varepsilon_{A} ; \operatorname{Ext}_{R}^{p}(M, R)\right) \cong \mathcal{H}^{q}\left(-E-\beta ; \operatorname{Ext}_{R}^{p}\left(M, \omega_{R}\right)\right)\left(-\varepsilon_{A}\right)$. We now determine the abutment by reversing the order of taking horizontal and vertical cohomology.

The natural projection from the total complex $\operatorname{Tot} \mathcal{K} .(E-\beta ; \mathcal{F} \cdot)$ to the EulerKoszul complex $\mathcal{K} .(E-\beta ; M)$ is a quasi-isomorphism because the rows of $\mathcal{K} .\left(E-\beta ; \mathcal{F}_{\cdot}\right)$ are resolutions for $M$ positioned in homological degree 0 . Since $\mathcal{H}_{k}(E-\beta ; M)$ is holonomic for all $k$ by Proposition [5.1 the complex $\operatorname{Hom}_{D}(\operatorname{Tot} \mathcal{K} .(E-\beta ; \mathcal{F} \cdot), D)$ has cohomology

$$
H^{p+q} \operatorname{Hom}_{D}(\operatorname{Tot} \mathcal{K} \cdot(E-\beta ; \mathcal{F} \cdot), D)=\operatorname{Ext}_{D}^{n}\left(\mathcal{H}_{p+q-n}(E-\beta ; M), D\right) .
$$

Applying the standard involution $\tau$ and the auto-equivalence (_- $)^{-}$yields

$$
H^{p+q} \mathbb{D}(\operatorname{Tot} \mathcal{K} \cdot(E-\beta ; \mathcal{F} \cdot))^{-}=\mathbb{D}\left(\mathcal{H}_{p+q-n}(E-\beta ; M)\right)^{-} .
$$

Remark 6.4. It follows from this spectral sequence that if $M$ is a Cohen-Macaulay toric module of dimension $d$, then $\mathcal{H}_{i}(E-\beta ; M)=0$ for all $i>0$ as then $\operatorname{Ext}_{R}^{n-d+i}\left(M, \omega_{R}\right)=0$ for all $i>0$. In the sequel we link the failure of $M$ to be Cohen-Macaulay to the appearance of nonvanishing $\mathcal{H}_{i}(E-\beta ; M)$ for suitable $\beta$ and $i>0$. 
Let $\mathfrak{m}$ be the maximal ideal $\left\langle\partial_{1}, \ldots, \partial_{n}\right\rangle$ of $R$; the pointed hypothesis on $A$ means that $\mathfrak{m}$ is the unique maximal $\mathbb{Z}^{d}$-graded ideal of $R$ and $\mathfrak{m} S_{A}$ is a (proper) maximal ideal of $S_{A}$. Given a $\mathbb{Z}^{d}$-graded $R$-module $N$, its local cohomology modules

$$
H_{\mathfrak{m}}^{i}(N)=\underset{t}{\lim } \operatorname{Ext}_{R}^{i}\left(R / \mathfrak{m}^{t}, N\right)
$$

supported at $\mathfrak{m}$ are $\mathbb{Z}^{d}$-graded. We refer to MS04 for details on the $\mathbb{Z}^{d}$-graded aspects of local cohomology. By [BH93, Section 3.5] there is a natural vector space isomorphism

$$
\operatorname{Ext}_{R}^{i}(N, R)_{\alpha} \cong \operatorname{Hom}_{\mathbb{C}}\left(H_{\mathfrak{m}}^{n-i}(N)_{-\alpha+\varepsilon_{A}}, \mathbb{C}\right)
$$

called $\mathbb{Z}^{d}$-graded local duality (see also Mil02 for more details on the $\mathbb{Z}^{d}$-graded aspects).

Definition 6.5. Fix a toric module $M$. A degree $\alpha \in \mathbb{Z}^{d}$ such that $H_{\mathfrak{m}}^{i}(M)_{-\alpha} \neq 0$ (note the minus sign in the subscript) for some $i \leq d-1$ is called a true exceptional degree of $M$. If $\beta \in \mathbb{C}^{d}$ lies in the Zariski closure of the set of true exceptional degrees of $M$, then $\beta$ is an exceptional quasi-degree of $M$.

Exactness of the Euler-Koszul complex can be expressed in terms of local cohomology.

Theorem 6.6. The Euler-Koszul homology $\mathcal{H}_{i}(E-\beta ; M)$ of a toric module $M$ over $R$ is nonzero for some $i \geq 1$ if and only if $\beta \in \mathbb{C}^{d}$ is an exceptional quasidegree of $M$. More precisely, if $k$ equals the smallest homological degree $i$ for which $-\beta \in \operatorname{qdeg}\left(H_{\mathfrak{m}}^{i}(M)\right)$, then $\mathcal{H}_{d-k}(E-\beta ; M)$ is holonomic of nonzero rank while $\mathcal{H}_{i}(E-\beta ; M)=0$ for $i>d-k$.

Proof. The module $M$ has dimension at most $d$, because this is true of every successive quotient in any toric filtration. Therefore, using notation as in Theorem 6.3. the modules $\operatorname{Ext}_{R}^{p}\left(M, \omega_{R}\right)$ can only be nonzero when $p \geq n-d$.

First suppose that $\beta$ is not an exceptional quasi-degree. This means precisely that $\beta$ lies outside of $q \operatorname{qdeg}\left(\operatorname{Ext}_{R}^{p}\left(M, \omega_{R}\right)\right)$ for all $p \neq n-d$ by local duality. Proposition 5.3 therefore implies that the only column of the spectral sequence page $E_{2}^{p, q}$ in Theorem 6.3 (note the $E+\beta$, not $E-\beta$ there) that can possibly be nonzero is column $p=n-d$. Furthermore, the highest possible row of a nonzero entry in this column is row $q=d$. Since the cohomology of the abutment is only nonzero when $p+q \geq n$, it follows that $\mathbb{D}\left(\mathcal{H}_{p+q-n}(E-\beta ; M)\right)^{-}$can only be nonzero when $p+q=n$. Applying the auto-equivalence $\left({ }_{-}\right)^{-}$and taking holonomic duals, we find that $\mathcal{H}_{i}(E-\beta ; M)$ can only be nonzero when $i=0$.

Now suppose that $\beta$ is an exceptional quasi-degree. Let $k$ be the smallest cohomological degree $i$ such that $\beta \in-\operatorname{qdeg}\left(H_{\mathfrak{m}}^{i}(M)\right)=\operatorname{qdeg}\left(\operatorname{Ext}_{R}^{n-i}\left(M, \omega_{R}\right)\right)$. By Proposition 5.3 and Lemma $6.1 \mathcal{H}^{d}\left(E+\beta ; \operatorname{Ext}_{R}^{n-k}\left(M, \omega_{R}\right)\right)\left(-\varepsilon_{A}\right)$ is nonzero. Moreover, all columns to the right of column $n-k$ and all rows above row $d$ in the spectral sequence page $E_{2}^{p, q}$ of Theorem 6.3 are zero. Hence

$$
\mathcal{H}^{d}\left(E+\beta ; \operatorname{Ext}_{R}^{n-k}\left(M, \omega_{R}\right)\right)\left(-\varepsilon_{A}\right)=\mathbb{D}\left(\mathcal{H}_{d-k}(E-\beta ; M)\right)^{-}
$$

is by Proposition 5.1 a holonomic module of nonzero rank. After applying the autoequivalence $\left({ }_{-}\right)^{-}$and taking holonomic duals, we find that $\mathcal{H}_{d-k}(E-\beta ; M)$ is a holonomic module of nonzero rank, and the highest nonzero Euler-Koszul homology of $M$. 


\section{Global Euler-Koszul homology as a holonomic family}

In this section we explore the interactions of the Euler-Koszul functor with our notion of holonomic family. The main result, Theorem 7.5 , is that the family of $D$ modules $\mathcal{H}_{0}(E-\beta ; \mathcal{M})$ for varying $\beta \in \mathbb{C}^{d}$ constitutes a holonomic family over $\mathbb{C}^{d}$.

Resume the notation from Example 2.2 and Definition 4.2 . If we give all of the variables $b=b_{1}, \ldots, b_{d}$ degree zero, then the polynomial ring $D[b]$ over the Weyl algebra $D$ is $\mathbb{Z}^{d}$-graded, as is its commutative subalgebra $R[b]$.

Definition 7.1. Let $\mathcal{M}$ be a $\mathbb{Z}^{d}$-graded $D[b]$-module. The global Euler endomorphisms of $\mathcal{M}$ are the commuting endomorphisms $E_{1}-b_{1}, \ldots, E_{d}-b_{d}$, the $i^{\text {th }}$ of which acts by

$$
E_{i}-b_{i}: m \mapsto\left(E_{i}-b_{i}-\alpha_{i}\right) m \text { whenever } m \in \mathcal{M}_{\alpha} .
$$

Each $\mathbb{Z}^{d}$-graded $R$-module $N$ yields a global Euler-Koszul complex of left $D[b]$ modules,

$$
\mathcal{K} \cdot(E-b ; N)=\mathcal{K} \cdot\left(E-b ; D[b] \otimes_{R} N\right)
$$

with $D[b]$-linear homomorphisms and homology $\mathcal{H}_{i}(E-b ; N)=H_{i} \mathcal{K} \cdot(E-b ; N)$.

For any fixed $\beta \in \mathbb{C}^{d}$, the zeroth Euler-Koszul homology $\mathcal{H}_{0}(E-\beta ; N)$ of $N$ can be recovered as a fiber (in the sense of Section 2) of the zeroth global Euler-Koszul homology $\mathcal{H}_{0}(E-b ; N)$ of $N$. The precise statement, as follows, is immediate from the definitions.

Lemma 7.2. Suppose that $N$ is a $\mathbb{Z}^{d}$-graded $R$-module, and that $\beta \in \mathbb{C}^{d}$. The fiber of $\mathcal{M}=\mathcal{H}_{0}(E-b ; N)$ over $\beta$ is $\mathcal{M}_{\beta}=\mathcal{H}_{0}(E-\beta ; N)$.

In view of Lemma 7.2 , to prove holonomicity for the family $\mathcal{H}_{0}(E-b ; M)$ determined by a toric $R$-module $M$ we need to establish the coherence condition in Definition 2.1. For this, we shall use the criterion in Proposition 3.4. But before we can do so, we need to know that Euler operators form sequences of parameters on the quotients of polynomial rings by certain initial ideals of toric ideals. We will get a handle on these initial ideals via their minimal primes. For notation, consider the polynomial ring $\mathbb{C}[\xi](x)$ with its usual $\mathbb{Z}$-grading, so each variable in the list $\xi=\xi_{1}, \ldots, \xi_{n}$ has degree 1 . A minimal prime of a finitely generated $\mathbb{C}[\xi](x)$-module $N$ is a prime minimal among those containing the annihilator of $N$.

Lemma 7.3. Let $N$ be a finitely generated $\mathbb{Z}$-graded $\mathbb{C}[\xi](x)$-module, and let $\mathbf{y}$ be a sequence of $\mathbb{Z}$-graded elements in $\mathbb{C}[\xi](x)$. If $\mathbb{C}[\xi](x) /(\langle\mathbf{y}\rangle+\mathfrak{p})$ is finite-dimensional as a vector space over $\mathbb{C}(x)$ for every minimal prime $\mathfrak{p}$ of $N$, then so is $N / \mathbf{y} N$.

Proof. Let $I \subseteq \mathbb{C}[\xi](x)$ be the annihilator of $N$. As $N$ is finitely generated over $\mathbb{C}[\xi](x) / I$, it is enough to show that $\langle\mathbf{y}\rangle+I$ contains a power of the $\mathbb{Z}$-graded maximal ideal $\mathfrak{m}=\langle\xi\rangle$.

Suppose that $I$ has radical $J$. Then if $\mathfrak{m}^{s} \subseteq\langle\mathbf{y}\rangle+J$, then $\mathfrak{m}^{r s} \subseteq(\langle\mathbf{y}\rangle+J)^{r}$, and so $\mathfrak{m}^{r s} \subseteq(\langle\mathbf{y}\rangle+J)^{r} \subseteq\langle\mathbf{y}\rangle+J^{r} \subseteq\langle\mathbf{y}\rangle+I$ for some $r$. Since the minimal primes of $I$ and $J$ are identical it is sufficient to consider $J$ instead of $I$.

Now let $J=\mathfrak{p}_{1} \cap \cdots \cap \mathfrak{p}_{m}$ be a primary decomposition where of course each $\mathfrak{p}_{j}$ is prime. Then $\langle\mathbf{y}\rangle+J=\langle\mathbf{y}\rangle+\left(\mathfrak{p}_{1} \cap \mathfrak{p}_{2} \cap \cdots \cap \mathfrak{p}_{m}\right)$ contains the product $\left(\langle\mathbf{y}\rangle+\mathfrak{p}_{1}\right) \cdots\left(\langle\mathbf{y}\rangle+\mathfrak{p}_{m}\right)$. Each of the factors $\left(\langle\mathbf{y}\rangle+\mathfrak{p}_{j}\right)$ contains a power of $\mathfrak{m}$ by the hypothesis, so $J+\langle\mathbf{y}\rangle$ contains the product of these powers of $\mathfrak{m}$. 
We now return to the order filtration on the Weyl algebra $D$ and the associated graded ring $\mathbb{C}[x, \xi]$ (see Section[3). This filtration, when restricted to the subring $R$ of $D$, gives an associated graded ring $\mathbb{C}[\xi]$. It induces on $R$ the partial ordering by total degree in which the initial form of a polynomial $f \in R$ is the $\operatorname{sum} \operatorname{in}(f) \in \mathbb{C}[\xi]$ of all terms of highest total degree, with the variables $\partial_{i}$ changed to $\xi_{i}$. The initial ideal of any ideal $J \subseteq R$ is the ideal $\operatorname{in}(J)=\langle\operatorname{in}(f) \mid f \in J\rangle$ generated by the initial forms of all polynomials in $J$.

In the following proposition, we consider the ideal $I_{A}^{F}$ from Notation 4.4. Let $A x \xi \subset \mathbb{C}[\xi](x)$ be the list in $\left(E_{1}\right), \ldots$, in $\left(E_{d}\right)$ obtained from $E_{1}, \ldots, E_{d}$ by replacing each $\partial_{i}$ with $\xi_{i}$.

Proposition 7.4. If $\operatorname{in}\left(I_{A}^{F}\right) \subseteq \mathbb{C}[\xi]$ is the initial ideal of $I_{A}^{F}$ in the above sense, then the $\mathbb{C}(x)$-vector space $\mathbb{C}[\xi](x) /\left\langle\operatorname{in}\left(I_{A}^{F}\right), A x \xi\right\rangle$ is finite-dimensional over $\mathbb{C}(x)$.

Proof. This statement immediately reduces to the case where $I_{A}^{F}=I_{A}$, after first replacing $\mathbb{C}[\xi](x)$ with $\mathbb{C}\left[\xi_{j} \mid a_{j} \in F\right]\left(x_{j} \mid a_{j} \in F\right)$, and then using any matrix $A_{F}^{\prime}$ composed of $\operatorname{dim}(F)$ many linearly independent rows of $F$ to play the role of $A$. We wish to apply Lemma 7.3, so we need to describe the minimal primes of $\mathbb{C}[\xi] / \operatorname{in}\left(I_{A}\right)$.

Define the $(d+1) \times(n+1)$ matrix $\hat{A}$ by placing a row $(1, \ldots, 1)$ across the top of $A$, and subsequently adding a leftmost column $\hat{a}_{0}=(1,0,0, \ldots, 0)$. If $\xi_{0}$ is a new variable and $\hat{\xi}=\left\{\xi_{0}\right\} \cup \xi$, then $\mathbb{C}[\xi] / \operatorname{in}\left(I_{A}\right) \cong \mathbb{C}[\hat{\xi}] /\left\langle I_{\hat{A}}, \xi_{0}\right\rangle$. Since the ideal $\left\langle I_{\hat{A}}, \xi_{0}\right\rangle$ is $\mathbb{Z} \hat{A}$-graded, every minimal prime of $\mathbb{C}[\xi] / \operatorname{in}\left(I_{A}\right)$ is the image in $\mathbb{C}[\xi]=\mathbb{C}[\hat{\xi}] /\left\langle\xi_{0}\right\rangle$ of a prime ideal $I_{\hat{A}} \subset \mathbb{C}[\hat{\xi}]$ for some face $\hat{F}$ of $\mathbb{N} \hat{A}$ with $\xi_{0} \in I_{\hat{A}}^{\hat{F}}$, where $I_{\hat{A}}^{\hat{F}_{\text {}}}$ is the $\xi$-analogue of $I_{A}^{F}$ for $\hat{A}$ and $\hat{F}$.

Thus we only need that for all faces $\hat{F}$ of $\mathbb{N} \hat{A}$ with $\xi_{0} \in I_{\hat{A}}^{\hat{F}}$, the ring $\mathbb{C}[\hat{\xi}](\hat{x}) /$ $\left\langle I_{\hat{A}}^{\hat{F}}, A x \xi\right\rangle$ has finite dimension as a $\mathbb{C}(\hat{x})$-vector space. Pick one such $\hat{F}$. Then $\hat{a}_{0} \notin \hat{F}$, since $\xi_{0} \in I_{\hat{A}}^{\hat{F}_{\text {. }}}$. Hence the columns of $\hat{F}$ correspond to a face $F$ of $A$ whose $\mathbb{C}$-affine span does not contain the origin; indeed, there is a bijection between the faces of $\hat{A}$ not containing $\hat{a}_{0}$ and the faces of [the convex hull of the origin and $A$ ] whose affine span does not contain the origin.

We infer that the columns of $A$ constituting $F$ lie on a hyperplane in $\mathbb{C}^{d}$ off the origin. Therefore the vector $(1, \ldots, 1) \in \mathbb{C}^{|F|}$ lies in the row span of $F$. This implies that

$$
\begin{aligned}
\mathbb{C}[\hat{\xi}](\hat{x}) /\left\langle I_{\hat{A}}^{\hat{F}}, A x \xi\right\rangle & =\mathbb{C}[\hat{\xi}](\hat{x}) /\left\langle I_{\hat{\hat{A}}}^{\hat{F}}, F x \xi\right\rangle \\
& =\mathbb{C}[\hat{\xi}](\hat{x}) /\left\langle I_{\hat{\hat{A}}}, \hat{F} x \xi\right\rangle \\
& =\mathbb{C}[\hat{\xi}](\hat{x}) /\left\langle I_{\hat{\hat{A}}}^{\hat{\hat{H}}}, \hat{A} \hat{x} \hat{\xi}\right\rangle
\end{aligned}
$$

where we use $F x \xi=\left\{\operatorname{in}\left(E_{1}^{F}\right), \ldots, \operatorname{in}\left(E_{d}^{F}\right)\right\}, \hat{F} x \xi=\left\{\sum_{j \in F} x_{j} \xi_{j}, \operatorname{in}\left(E_{1}^{F}\right), \ldots, \operatorname{in}\left(E_{d}^{F}\right)\right\}$, and $\hat{A} \hat{x} \hat{\xi}=\left\{\sum_{j=0}^{n} x_{j} \xi_{j}\right.$, in $\left(E_{1}\right), \ldots$, in $\left.\left(E_{d}\right)\right\}$.

The proof has thus been reduced to the projective situation. This case of the proposition follows from the proof of [Ado94, Theorem 3.9], which proceeds by showing precisely that in the projective situation the Krull dimension of $\mathbb{C}[x, \xi] /$ (in $\left.\left(I_{A}\right)+\langle A x \xi\rangle\right)$ equals $n$.

Theorem 7.5. For any toric $R$-module $M$, the sheaf $\tilde{\mathcal{M}}$ on $\mathbb{C}^{d}$ whose global section module is $\mathcal{M}=\mathcal{H}_{0}(E-b ; M)$ constitutes a holonomic family over $\mathbb{C}^{d}$; in other words, $\mathcal{M}_{\beta}=\mathcal{H}_{0}(E-\beta ; M)$ is holonomic for all $\beta \in \mathbb{C}^{d}$, and $\mathcal{M}(x)$ is finitely generated over $\mathbb{C}[b](x)$. 
Proof. That the fibers $\mathcal{M}_{\beta}=\mathcal{H}_{0}(E-\beta ; M)$ are holonomic modules is a consequence of Lemma 7.2 and Proposition 5.1 .

For the coherence condition, suppose first that $M=S_{F}$ for some face $F$. Using order filtrations as above, the graded $\mathbb{C}[b](x)$-module associated to $\mathcal{M}(x)$ is a quotient of $N=\mathbb{C}[\xi][b](x) /\left\langle\operatorname{in}\left(I_{A}^{F}\right), A x \xi\right\rangle$ for in $\left(I_{A}^{F}\right)+\langle A x \xi\rangle=\operatorname{in}\left(I_{A}^{F}\right)+\operatorname{in}(E-b) \subseteq$ in $\left(\left\langle I_{A}^{F}, E-b\right\rangle\right)$. As the generators for in $\left(I_{A}^{F}\right)$ and $A x \xi$ do not involve $b$, Proposition 7.4 implies that $N$ is finitely generated over $\mathbb{C}[b](x)$. Hence $\mathcal{M}(x)$ is finitely generated by Proposition 3.4 .

Now let $M$ be any toric $R$-module. For $\alpha \in \mathbb{Z}^{d}$ and with $b_{i}^{\prime}=b_{i}-\alpha_{i}$ the isomorphism

$$
\mathcal{H}_{0}\left(E-b^{\prime} ; S_{F}\right)(\alpha)=\mathcal{H}_{0}\left(E-b+\alpha ; S_{F}\right)(\alpha)=\mathcal{H}_{0}\left(E-b ; S_{F}(\alpha)\right)
$$

together with the independence of both in $\left(I_{A}^{F}\right)$ and $A x \xi$ of $b$ proves the theorem if $M$ has toric length $\ell=1$. For $\ell>1$, use the short exact sequence $M_{1} \hookrightarrow M \rightarrow M / M_{1}$ from a toric filtration. The resulting long exact sequence of global Euler-Koszul homology $\mathcal{H} .\left(E-b ;_{-}\right)$tensored with the flat module $\mathbb{C}(x)$ places the module $\mathcal{H}_{0}(E-b ; M)(x)$ between the two modules $\mathcal{H}_{0}\left(E-b ; M_{1}\right)(x)$ and $\mathcal{H}_{0}\left(E-b ; M / M_{1}\right)(x)$, both of which are finitely generated by induction on $\ell$.

\section{ISOMORPHISM OF THE TWO HOMOLOGY THEORIES}

Let $B$ be a reduced parameter scheme and suppose $\beta \in B_{\mathbb{C}}$ is determined by a regular sequence $\mathbf{y}$ in $\Gamma\left(B, \mathcal{O}_{B}\right)$. In Corollary 3.3 we found that the commutative Koszul complex $K \cdot(\mathbf{y}, \mathcal{M}(x))$ detects rank-jumps in holonomic families $\mathcal{M}$ of $D$ modules at $\beta$. In the special case of generalized hypergeometric systems associated to toric modules, where in particular $B=\mathbb{C}^{d}$, we also found in Theorem 6.6 that the noncommutative Euler-Koszul complex $\mathcal{K} .(E-\beta ; M)$ detects the quasi-degrees where local cohomology of the toric $R$-module $M$ is nonvanishing in cohomological degree less than $d$. In this section we prove that if $\mathcal{M}=\mathcal{H}_{0}(E-b ; M)$ is defined through the toric module $M$, then these two complexes are isomorphic in the derived category, and in particular have the same homology.

We begin by noting that the global Euler-Koszul complex can be interpreted as a collection of commutative Koszul complexes, similarly to Lemma 4.3.

Lemma 8.1. Let $N$ be a $\mathbb{Z}^{d}$-graded $R$-module and $\alpha \in \mathbb{Z}^{d}$. The $\mathbb{Z}^{d}$-graded degree $\alpha$ part $\mathcal{K} .(E-b ; N)_{\alpha}$ of the global Euler-Koszul complex agrees with the ordinary Koszul complex $K \cdot\left(E-b-\alpha ;\left(D[b] \otimes_{R} N\right)_{\alpha}\right)$ constructed over the polynomial ring $\Theta[b] \subseteq D[b]$. That is,

$$
\mathcal{K} \cdot(E-b ; N)=\bigoplus_{\alpha \in \mathbb{Z}^{d}} K \cdot\left(E-b-\alpha ;\left(D[b] \otimes_{R} N\right)_{\alpha}\right),
$$

the right-hand side being a direct sum of ordinary Koszul complexes of $\Theta[b]$-modules.

Recall from Theorem 7.5 that $\mathcal{H}_{0}(E-b ; M)$ represents a holonomic family whenever $M$ is a toric $R$-module. The following statement links the two homological theories that we have studied: Euler-Koszul homology and Koszul homology of holonomic families.

Theorem 8.2. Let $M$ be a toric $R$-module, and consider the holonomic family on $\mathbb{C}^{d}$ with sections $\mathcal{M}=\mathcal{H}_{0}(E-b ; M)$. For each parameter vector $\beta \in \mathbb{C}^{d}$, the 
Euler-Koszul homology of $M$ and the ordinary Koszul homology of $\mathcal{M}$ over $\mathbb{C}[b]$ are isomorphic:

$$
\mathcal{H}_{i}(E-\beta ; M) \cong H_{i}(b-\beta ; \mathcal{M}) .
$$

Proof. Write $\mathcal{K}^{E}=\mathcal{K} .(E-b ; M)$ for the global Euler-Koszul complex, thought of as a column pointing downward with its bottom at row 0 , and let $K^{b}=K \cdot(b-\beta ; \mathbb{C}[b])$ be the ordinary Koszul complex on $b-\beta$, thought of as a row pointing leftward toward its end at column 0 . Consider the double complex $K^{b} \otimes_{\mathbb{C}[b]} \mathcal{K}^{E}$.

Taking horizontal $\left(K^{b}\right)$-homology first leaves only one column, namely the leftmost column, and that column is the Euler-Koszul complex $\mathcal{K} \cdot(E-\beta ; M)$. It follows that $H \cdot \operatorname{Tot}\left(K_{\bullet}^{b} \otimes \mathcal{K}_{\bullet}^{E}\right) \cong \mathcal{H} \cdot(E-\beta ; M)$.

Now we need to check that $H \cdot \operatorname{Tot}\left(K_{\bullet}^{b} \otimes \mathcal{K}_{\bullet}^{E}\right) \cong H \cdot(b-\beta ; \mathcal{M})$. For this it is enough to show that taking the vertical $\left(\mathcal{K}^{E}\right)$-homology of the double complex leaves only one row (i.e., the bottom row), for then that row is the Koszul complex $K^{b}$. tensored over $\mathbb{C}[b]$ with the global Euler-Koszul homology $\mathcal{M}=\mathcal{H}_{0}(E-b ; M)$.

All the homomorphisms in $K^{b} \otimes \mathcal{K}^{E}$ are $\mathbb{Z}^{d}$-graded, so it suffices to check the acyclicity of the vertical differential separately on each $\mathbb{Z}^{d}$-graded component. But $\left(D[b] \otimes_{R} M\right)_{\alpha}=\left(D \otimes_{R} M\right)_{\alpha} \otimes_{\mathbb{C}} \mathbb{C}[b]$, so $\left(b_{1}-\left(E_{1}-\alpha_{1}\right), \ldots, b_{d}-\left(E_{d}-\alpha_{d}\right)\right)=$ $-(E-b-\alpha)$ is a regular sequence in $\Theta[b]$ on the $\Theta[b]$-module $\left(D[b] \otimes_{R} M\right)_{\alpha}$. Now use Lemma 8.1 .

\section{COMBinatorics OF HYPERGEOMETRIC RANKS}

This section contains our results on our motivating problem: a geometric description of the rank-jumping locus for generalized hypergeometric systems (Theorem 9.1 and Corollary 9.4), and a characterization of Cohen-Macaulayness for semigroup rings through the absence of rank-jumps (Corollary 9.2).

Theorem 9.1. The rank-jumping parameters $\beta \in \mathbb{C}^{d}$ for the hypergeometric holonomic family $\mathcal{H}_{0}(E-b ; M)$ of a toric module $M$ are the exceptional quasi-degrees of $M$.

Proof. By Theorem 6.6, a parameter $\beta$ is an exceptional quasi-degree of $M$ if and only if the Euler-Koszul homology $\mathcal{H}_{i}(E-\beta ; M)$ is of nonzero rank for some $i>0$. This occurs if and only if the homology $H_{i}(b-\beta ; \mathcal{M})$ of the hypergeometric holonomic family $\mathcal{M}=\mathcal{H}_{0}(E-b ; M)$ is holonomic of nonzero rank for some $i>0$, by Theorem 8.2. The nonzero rank condition ensures that the nonvanishing of $H_{i}(b-\beta ; \mathcal{M})$ is equivalent to its nonvanishing after tensoring with $\mathbb{C}(x)$ over $\mathbb{C}[x]$. But $\mathbb{C}(x) \otimes_{\mathbb{C}[x]} H_{i}(b-\beta ; \mathcal{M})=H_{i}(b-\beta ; \mathcal{M}(x))$ by exactness of localization, and this Koszul homology is nonzero if and only if $\beta$ is rank-jumping by Corollary 3.3 .

For a finitely generated $R$-module, the vanishing of $H_{\mathfrak{m}}^{i}(M)$ for all $i<d$ is equivalent to $M$ having depth $d$. This immediately implies the following.

Corollary 9.2. The holonomic family $\mathcal{M}_{\beta}^{A}=\mathcal{H}_{0}\left(E-\beta ; S_{A}\right)$ has a nonempty set of rank-jumping parameters $\beta \in \mathbb{C}^{d}$ if and only if $S_{A}=R / I_{A}$ fails to be CohenMacaulay.

Remark 9.3. Corollary 9.2 assumes - as we do throughout this paper (see Section (4) - that $A$ is pointed. When $A$ is not pointed, some of the $\partial_{i}$ are units in $S_{A}$, so the statement of (for example) Theorem 6.6 must be adapted, since $H_{\mathfrak{m}}^{0}\left({ }_{-}\right)$is then the zero functor. Although we suspect that Corollary 9.2 holds when $A$ is not 
pointed, it does not follow immediately from the pointed case. In more detail, we are willing to believe that the entire problem reduces immediately to the pointed case when $\mathbb{N} A$ splits as (a pointed affine semigroup) $\times$ (units); but in general, the units of $\mathbb{N} A$ can have finite index in the units of the normalization, so $\mathbb{N} A$ modulo its units is not a pointed affine semigroup, as the associated group has torsion.

Not only is the rank-jump locus closed in $\mathbb{C}^{d}$ but it has a nice geometric structure.

Corollary 9.4. The set of rank-jumping parameters is a finite union of translates of linear subspaces $\mathbb{C} F$ generated by faces $F$ of $A$. A translate of $\mathbb{C} F$ appears if and only if the prime ideal $I_{A}^{F}$ lies in the support of $\operatorname{Ext}_{R}^{n-i}(M, R)$ for some $i \leq d-1$.

Proof. The graded Matlis dual of the local cohomology module $H_{\mathfrak{m}}^{i}(M)$ is, by local duality, a $\mathbb{Z}^{d}$-graded translate of $\operatorname{Ext}_{R}^{n-i}(M, R)$. Hence the rank-jumping parameters form a $\mathbb{Z}^{d}$-graded translate of the $\operatorname{set} \mathrm{qdeg}\left(\bigoplus_{i=0}^{d-1} \operatorname{Ext}_{R}^{n-i}(M, R)\right)$.

Porism 9.5. The set of exceptional parameters for a GKZ hypergeometric system $\mathcal{M}_{\beta}^{A}=\mathcal{H}_{0}\left(E-\beta ; S_{A}\right)$ has codimension at least 2 in $\mathbb{C}^{d}$.

Proof. Set $S=S_{A}$. By Corollary 9.4, we need $\operatorname{Ext}_{R}^{n-i}(S, R)_{\mathfrak{p}}=0$ for all primes $\mathfrak{p}$ of dimension $d-1$ and all $i \leq d-1$. For such primes, $R_{\mathfrak{p}}$ is regular and local of dimension $n-d+1$, so $\operatorname{Ext}_{R}^{n-i}(S, R)_{\mathfrak{p}}=\operatorname{Ext}_{R_{\mathfrak{p}}}^{n-i}\left(S_{\mathfrak{p}}, R_{\mathfrak{p}}\right)=0$ unless $i \geq d-1$. As $\operatorname{Ext}_{R}^{n-d+1}(S, R)_{\mathfrak{p}}$ is Matlis dual over $R_{\mathfrak{p}}$ to $H_{\mathfrak{p} R_{\mathfrak{p}}}^{0}\left(S_{\mathfrak{p}}\right)$, which vanishes because $S$ is a domain, we are done.

Remark 9.6. Corollary 9.4, and more generally Theorem 2.6. indicates that the dimensions of the holomorphic solution spaces of hypergeometric systems $\mathcal{M}_{\beta}^{A}$ behave quite tamely as functions of $\beta$. However, the methods in this article do not consider the variation of the holomorphic solutions themselves (cf. Remark 2.8), which could in principle be worse. In fact, work of Saito Sai01 implies that the isomorphism classes of $A$-hypergeometric systems $\mathcal{M}_{\beta}^{A}$ do not vary at all algebraically with $\beta$. The question therefore remains whether the holomorphic solution space of a hypergeometric system $\mathcal{M}_{\beta}^{A}$ varies as a function of $\beta$ more like the rank of $\mathcal{M}_{\beta}^{A}$ or more like the isomorphism class of $\mathcal{M}_{\beta}^{A}$. This problem is important for research toward constructing explicit solutions to $A$-hypergeometric systems.

\section{ACKNOWLEDGMENTS}

The equivalence of hypergeometric rank constancy and toric Cohen-Macaulayness was first conjectured by Bernd Sturmfels, who divulged it in talks and openproblem sessions. He was also the first to mention local cohomology in connection with rank-jumps, although not in the precise form that appears in this article. His encouragement and advice have been invaluable to us throughout this project.

The occasions when all three authors of this article were in the same place at the same time have been rare, and all the more appreciated for that. We would like to thank the organizers of the Joint International Meeting of the AMS and RSME in Sevilla, Spain, for providing us with one such opportunity in the Summer of 2003. LFM and UW are especially grateful to Francisco Castro-Jiménez, Jose María Ucha, and María Isabel Hartillo Hermoso, who were our gracious hosts when we stayed 
in Sevilla for a week after that conference. All three authors also intersected at MSRI. We are indebted to this institute for the friendly and stimulating research atmosphere it provides.

Finally, we thank Razvan Veliche and the referee for particularly careful readings.

\section{REFERENCES}

[Ado94] Alan Adolphson, Hypergeometric functions and rings generated by monomials, Duke Math. J. 73 (1994), no. 2, 269-290. MR.1262208 (96c:33020)

[Ado99] - Higher solutions of hypergeometric systems and Dwork cohomology, Rend. Sem. Mat. Univ. Padova 101 (1999), 179-190. MR1705287 (2001b:14032)

[BH93] Winfried Bruns and Jürgen Herzog, Cohen-Macaulay rings, Cambridge Studies in Advanced Mathematics, vol. 39, Cambridge University Press, Cambridge, 1993. MR1251956 (95h:13020)

[Bjö79] J.-E. Björk, Rings of differential operators, North-Holland Mathematical Library, vol. 21, North-Holland Publishing Co., Amsterdam, 1979. MR.0549189 (82g:32013)

[CDD99] Eduardo Cattani, Carlos D'Andrea, and Alicia Dickenstein, The A-hypergeometric system associated with a monomial curve, Duke Math. J. 99 (1999), no. 2, 179-207. MR.1708034 (2001f:33018)

[CDS01] Eduardo Cattani, Alicia Dickenstein, and Bernd Sturmfels, Rational hypergeometric functions, Compositio Math. 128 (2001), no. 2, 217-239. MR1850183 (2003f:33016)

[CK99] David Cox and Sheldon Katz, Mirror symmetry and algebraic geometry, Amer. Math. Soc., Providence, RI, 1999. MR1677117 (2000d:14048)

[Eis95] David Eisenbud, Commutative algebra, with a view toward algebraic geometry, Graduate Texts in Mathematics, vol. 150, Springer-Verlag, New York, 1995. MR1322960 (97a:13001)

[GGZ87] I. M. Gel'fand, M. I. Graev, and A. V. Zelevinskiř, Holonomic systems of equations and series of hypergeometric type, Dokl. Akad. Nauk SSSR 295 (1987), no. 1, 14-19. MR0902936 (88j:58118)

[GKZ89] I. M. Gel'fand, A. V. Zelevinskiı̌, and M. M. Kapranov, Hypergeometric functions and toric varieties, Funktsional. Anal. i Prilozhen. 23 (1989), no. 2, 12-26. Correction in ibid, 27 (1993), no. 4, 91. MR.1011353 (90m:22025) MR.1264328(95a:22010)

[Har77] Robin Hartshorne, Algebraic geometry, Graduate Texts in Mathematics, vol. 52, Springer-Verlag, New York, 1977. MR0463157 (57:3116)

[Hot98] Ryoshi Hotta, Equivariant D-modules, 1998. arXiv:math.RT/9805021

[Mat01] Laura Felicia Matusevich, Rank jumps in codimension 2 A-hypergeometric systems, J. Symbolic Comput. 32 (2001), no. 6, 619-641, Effective methods in rings of differential operators. MR 1866707 (2003f:33017)

[Mat03] Exceptional parameters for generic A-hypergeometric systems, Int. Math. Res. Not. (2003), no. 22, 1225-1248. MR 1967406 (2004b:16039)

[MM05] Laura Felicia Matusevich and Ezra Miller, Combinatorics of rank jumps in simplicial hypergeometric systems, Proc. Amer. Math. Soc., to appear, 2005. arXiv:math. AC/0402071

[MW04] Laura Felicia Matusevich and Uli Walther, Arbitrary rank jumps for A-hypergeometric systems through Laurent polynomials, 2004. arXiv:math.CO/0404183

[Mil02] Ezra Miller, Graded Greenlees-May duality and the Čech hull, Local cohomology and its applications (Guanajuato, 1999), Lecture Notes in Pure and Appl. Math., vol. 226, Dekker, New York, 2002, pp. 233-253. MR1888202 (2004b:13019)

[MS04] Ezra Miller and Bernd Sturmfels, Combinatorial commutative algebra, Graduate Texts in Mathematics Vol. 227, Springer-Verlag, New York, 2004. MR2110098

[Sai01] Mutsumi Saito, Isomorphism classes of A-hypergeometric systems, Compositio Math. 128 (2001), no. 3, 323-338. MR.1858340 (2003f:33019)

[Sai02] Mutsumi Saito, Logarithm-free A-hypergeometric series, Duke Math. J. 115 (2002), no. 1, 53-73. MR1932325 (2004f:16041)

[SST00] Mutsumi Saito, Bernd Sturmfels, and Nobuki Takayama, Gröbner deformations of hypergeometric differential equations, Algorithms and Computation in Mathematics, vol. 6, Springer-Verlag, Berlin, 2000. MR.1734566 (2001i:13036) 
[ST98] Bernd Sturmfels and Nobuki Takayama, Gröbner bases and hypergeometric functions, Gröbner bases and applications (Linz, 1998), London Math. Soc. Lecture Note Ser., vol. 251, Cambridge Univ. Press, Cambridge, 1998, pp. 246-258. MR.1708882 (2001c:33026)

Department of Mathematics, Harvard University, Cambridge, Massachusetts 02138

Current address: Department of Mathematics, University of Pennsylvania, Philadelphia, Pennsylvania 19104

E-mail address: lfm@math.upenn.edu

School of Mathematics, University of Minnesota, Minneapolis, Minnesota 55455

E-mail address: ezra@math.umn.edu

Department of Mathematics, Purdue University, West Lafayette, Indiana 47907

E-mail address: walther@math.purdue.edu 